\title{
Nonlinear electrostatic ion-acoustic "oscilliton" waves driven by charge non-neutrality effects
}

\author{
J. Z. G. Ma ${ }^{1}$, A. Hirose ${ }^{2}$, J.-P. St.-Maurice ${ }^{2}$, and W. Liu ${ }^{1}$ \\ ${ }^{1}$ Science and Academic Development, Canadian Space Agency, 6767, Route de l'Aéroport, Longueuil (Saint-Hubert), \\ Québec, J3Y 8Y9, Canada \\ ${ }^{2}$ Department of Physics and Engineering Physics, University of Saskatchewan, 116 Science Place, Saskatoon, Saskatchewan, \\ S7N 5E2, Canada
}

Received: 29 January 2010 - Revised: 8 December 2010 - Accepted: 15 December 2010 - Published: 11 January 2011

\begin{abstract}
Nonlinear "oscilliton" structures features a lowfrequency (LF) solitary envelope, the amplitude of which is modulated violently by superimposed high-frequency (HF) oscillations. We have studied the charge non-neutrality effects on the excitation of electrostatic ion-acoustic (IA) oscillitons. A two-fluid, warm plasma model is employed, and a set of nonlinear self-similar equations is solved in a cylindrical geometry. Under charge-neutrality conditions, three conventional IA structures (namely, sinusoidal, sawtooth, and spicky/bipolar) are obtained. By contrast, under charge nonneutrality conditions, oscilliton structures are excited, where the LF envelope is in the sound-wave (SW) mode, while the HF ingredients include the IA mode and the ion-Langmiur (IL) mode. The amplitudes of the SW wave are violently modulated by the IA oscillations, whereas the upward sides of the IA amplitudes are modulated by the IL oscillations of smaller amplitudes, and the downward sides are modulated by hybrid IA/IL oscillations. The nonlinear oscillitons are found to be dependent not only upon the input parameters (e.g., the Mach number, the Debye length, and the initial temperature of particles), but on initial conditions as well.
\end{abstract}

Keywords. Space plasma physics (Nonlinear phenomena)

\section{Introduction}

For more than 70 years, a salient waveform was captured in interplanetary spaces by numerous experiments: a lowfrequency (LF) envelope, the amplitude of which is modulated violently by high-frequency (HF) oscillations. In extraterrestrial spaces, for example, detections of such LFHF "symbiotic" packets began in the 1980s, when Gurnett (1985) reported the first measurement by the Voyager 1 and

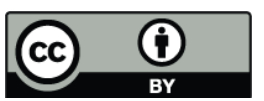

Correspondence to: J. Z. G. Ma (johnzg.ma@asc-csa.gc.ca)
2 spacecraft in the upstream of Jupiter's bow shock. The author suggested that the extraordinary structures are related to HF electron oscillations. In the vicinity of Io, the Galileo spacecraft recorded similar waveforms which were thought to be connected with ion-cyclotron (IC) waves (Kivelson et al., 1996). In the Martian upstream region, the Mars Global Surveyor confirmed that such structures also exist in the bow shock where the HF signature is exactly at the local proton IC frequency while the LF component is at about seven gyroperiods (see Mazelle et al., 2004, in details). In geospace, by contrast, such coexisting wave modes were found much earlier. A vast volume of experimental geomagnetic micropulsation data (1936-2000) exposes that the $0.4-2.2 \mathrm{~Hz}$ Pc1 HF modes superimpose upon the $1-250 \mathrm{mHz}$ Pc2-5 LF ones (Alpert, 2001). In the last 10-20 years, many highresolution satellites have introduced various HF-LF wavefield diagnoses beyond the scope of these frequencies in different plasma regions, such as Freja (Eriksson et al., 1994; Dovner et al., 1994; Pécseli et al., 1996), Polar (Cattell et al., 1998, 1999), FAST (McFadden et al., 1998; Pottelette et al., 1999), Viking (Tjulin et al., 2003, 2004), and Cluster (Santolik et al., 2003; Tjulin et al., 2003, 2004).

What on Earth is the Prime Mover that triggers such remarkable structures consisting of two or more phases, rather than respective simple waves we usually encounter? Usually, the fully developed linear MHD wave theory is prone to being used to account for the LF-HF excitations. As a matter of fact, linear MHD formulation does satisfactorily explain the geomagnetic pulsations created in the magnetosphere at frequencies of five branches Pc1-5 (see Alpert, 2001, and references therein for details): Pc1 is of the magnetospheric origin at the gyro-resonant IC frequency of magneto-active plasma ions, while others are of solar origin and transferred to the Earth's magnetosphere by the solar wind, driven by respective linear instabilities that are responsible for their generation.

Published by Copernicus Publications on behalf of the European Geosciences Union. 
Table 1. Some important space projects and relevant orders of typical plasma parameters measured in the ionospheric auroral F-layer by the Geodesic rocket (Burchill et al., 2004), in the topside ionosphere by the Freja satellite (Eriksson et al., 1994), in the auroral zone by the FAST satellite (Ergun, 1999), and in the magnetopause by the Cluster satellites (Tjulin et al., 2004). See Sect. 2 for definitions of parameters.

\begin{tabular}{ccccc}
\hline Vehicle & Geodesic & Freja & FAST & Cluster \\
\hline$m_{\mathrm{i}} / m_{\mathrm{p}}$ & 16 & $16 / 1$ & 1 & 1 \\
$B(\mathrm{mG})$ & 500 & 250 & 100 & 10 \\
$n_{0}\left(\mathrm{~cm}^{-3}\right)$ & $10^{6}$ & $10^{3}$ & 10 & 1 \\
$T_{\mathrm{i}}(\mathrm{eV})$ & 0.1 & 0.2 & 300 & 10 \\
$T_{\mathrm{e}}(\mathrm{eV})$ & 0.2 & 0.3 & 700 & 1 \\
$c_{\mathrm{S}}(\mathrm{km} / \mathrm{s})$ & 1 & $1.3 / 5.5$ & 260 & 10 \\
$c_{\mathrm{S}} B(\mathrm{mV} / \mathrm{m})$ & 50 & $33 / 140$ & 2600 & 10 \\
$\Omega_{\mathrm{i}}(\mathrm{krad} / \mathrm{s})$ & 0.3 & $0.15 / 2.4$ & 1 & 0.1 \\
$\omega_{\mathrm{pi}}(\mathrm{krad} / \mathrm{s})$ & 330 & $10 / 40$ & 4.16 & 1.3 \\
$\Omega_{\mathrm{e}}(\mathrm{krad} / \mathrm{s})$ & 8800 & 4400 & 1840 & 184 \\
$\omega_{\mathrm{pe}}(\mathrm{krad} / \mathrm{s})$ & 56550 & 1800 & 180 & 180 \\
$\omega_{\mathrm{LH}}(\mathrm{krad} / \mathrm{s})$ & 50 & $9 / 37$ & 4.14 & 0.9 \\
$\lambda_{\mathrm{De}}(\mathrm{m})$ & 0.003 & $0.13 / 0.14$ & 62 & 8 \\
$\rho_{\mathrm{i}}(\mathrm{m})$ & 3.3 & $8.7 / 2.3$ & 260 & 100 \\
$\xi_{\omega}\left(=\rho_{\mathrm{i}} / \lambda_{\mathrm{De}}\right)$ & 1100 & $67 / 17$ & 4 & 13 \\
\hline & & & &
\end{tabular}

Significantly, a nonlinear mechanism has been developed in the 2000s to account for the multi-frequency pulsations, other than Pc1-5, observed by most high-altitude satellites. The work was pioneered by Sauer et al. (2001) where the packets were named "oscillitons" (i.e., "oscillations" + "solitons"), followed by a series of subsequent publications, e.g., Sauer et al. (2002, 2003); Dubinin et al. (2002, 2003a, b, c, 2007); McKenzie et al. (2004); Cattaert and Verheest (2005), and Sydora et al. (2007). In view of nonlinear electromagnetic whistler modes, the authors found that oscillitons are excited in multi-ion plasmas in propagations parallel to, and supposedly also, oblique to a static, background magnetic field. The approaches integrated both analytical and numerical methods, and developed algorithms for effective simulations, aiming at case studies of instrumental spectra. The authors relied on multi-fluid models, along with a Sagdeev-potential description where necessary (e.g., Cattaert and Verheest, 2005), rather than on, independently, either KdV/Schrödinger (see, e.g., Pécseli, 1985) or Sagdeev-potential (see, e.g., Sagdeev and Galeev, 1969) methods. They not only provided a reinforced understanding of the existence of resonance conditions under which oscillitons appear (e.g., Verheest and Cattaert, 2005), but also offer a shortcut in comparative studies among similarly observed oscillitons by using data-fit modeling (e.g., Dubinin et al., 2007; Pottelette and Berthomier, 2009, and references therein). What is more, Kourakis and Shukla (2005) developed a generic methodological formulation for oscillitons by taking into account either the mutual modulation effect (due to the interaction between HF and LF modes) or the auto-modulation one (due to the self-interaction of a carrier mode). Both cold and warm fluid formulations are discussed and a multiple scale technique is applied.

Impressively, in studying large-amplitude, electromagnetic, nonlinear waves in electron-positron plasmas, (Verheest and Cattaert, 2004), Verheest et al. (2004) perceived a sensitive quality of the whistler oscillitons through numerical computations that had previously been unrecognized: at not too large amplitudes, oscillitons are very similar to single-frequency solitary envelopes obtained by solving the Schrödinger equation, either the charge neutrality condition is imposed as an a priori one or not. Intrigued by this study, we are curious about the role played by this condition in the excitation of conventional single-frequency solitary envelopes and multi-frequency oscilliton structures. To make it clear is beneficial to gain a deeper insight into the physics of the formation of the multi-frequency nonlinear structures.

As a matter of fact, the breakdown of the charge neutrality condition can lead to conspicuous effects in a plasma system: a slight divergence between positive and negative charge densities (caused by, e.g., background density perturbations, precipitating electron beams) produces a "strong" space-charge electric field. For example, in a cylindrical frame, a space-charge density of $n_{\mathrm{sc}} \sim 10^{-5}$ times smaller than the ambient plasma density could bring about a characteristic electric field $E_{\mathrm{c}}=100 \mathrm{mV} \mathrm{m}^{-1}$ at a characteristic radius $R_{\mathrm{c}}=10 \mathrm{~m}$, and thus cause an $\boldsymbol{E} \times \boldsymbol{B}$ drift of $2 \mathrm{~km} \mathrm{~s}^{-1}$ in an ambient magnetic field of $B \sim 0.5 \mathrm{G}$. This drift is higher than the ion thermal speed which is about $1 \mathrm{~km} / \mathrm{s}$ in ionospheric plasmas (this is why we say such a field is "strong"), and therefore produces noneligible consequences to particle kinetics and bulk properties as studied by, e.g., Cole (1971), Schunk and Walker (1972), St.-Maurice et al. (1976, 1994), Jørgensen (1978), St.-Maurice and Schunk (1979), Lockwood et al. (1987), Winkler et al. (1992), Gaimard et al. (1998), Ma and St.-Maurice (2008).

These studies show that the charge non-neutrality condition should also influence the excitation and the features of nonlinear structures due to the fact that nonlinear processes are determined by plasma dynamics. Encouraged by Verheest et al. (2004), we uncovered the three traditional, singlefrequency, solitary packets under charge neutrality conditions in both a Cartesian geometry (Ma and Hirose, 2009) and a cylindrical one (Ma, 2010). However, this condition may not be encountered at high altitudes above the ionospheric F-layer where multi-frequency waveforms are reported frequently, while it is encountered at low altitudes where though rockets are able to reach and few such waveforms are available. The reader is asked here to view several typical space projects listed in Table 1 along with key plasma parameters. The regions concerned are as follows: the ionospheric auroral F-layer by the GEODESIC rocket (Burchill et al., 2004), topside ionosphere by the Freja satellite (Eriksson et al., 1994), the auroral zone by the FAST satellite (Ergun, 
1999), and the magnetopause by the Cluster satellites (Tjulin et al., 2004). Section 2 gives the definitions of all parameters. The table shows that plasmas are very different in regions above the ionospheric F-layer from those below. An obvious consequence of this is about the validity of the chargeneutrality condition. For example, both satellite data and simulation results exhibit that the spatial scales of nonlinear waves are about several ion gyro-radii $\left(\rho_{\mathrm{i}}\right.$; see, e.g., Ergun et al., 1998a, b). For a plasma system with an electron Debye length $\left(\lambda_{\mathrm{De}}\right)$, it is charge-neutralized when $\rho_{\mathrm{i}} \gg \lambda_{\mathrm{De}}$, or, $\xi_{\omega}=\rho_{\mathrm{i}} / \lambda_{\mathrm{De}} \gg 1$, as given by the Geodesic rocket data $\xi_{\omega}>1000$. In this case, electron and ion densities can be approximated as $n_{\mathrm{i}} \approx n_{\mathrm{e}}$ (here $n_{\mathrm{i}}$ and $n_{\mathrm{e}}$ represent ion and electron densities, respectively). On the contrary, it is at a charge non-neutrality state, just as given by the satellite data $\xi_{\omega}<100$. In this case, a space-charge density $n_{\mathrm{sc}}=n_{\mathrm{i}}-n_{\mathrm{e}}$ exists inevitably.

Because linear MHD wave mechanisms under chargeneutrality conditions explain the production of Pc1-5 multiwave pulsations, we naturally consider that a nonlinear process, induced by the charge non-neutrality condition, should have a connection to the activation of the multi-ingredient oscilliton waveforms. Previous authors (e.g., Verheest et al., 2004) showed that this process is irrelevant of either electrostatic or electromagnetic modes, while other authors (e.g., Ma, 2010) confirmed that the charge-neutrality is dedicated only to single-wave nonlinear structures. We thus expand the original meaning of the beautiful word, "oscillitons", to describe a kind of nonlinear multi-frequency waveform structure of either electromagnetic or electrostatic origin. Unfortunately, contrary to the extensive studies on electromagnetic "oscillitons" mentioned above, little work has been reported on the electrostatic "oscillitons". We are introducing the first part of our work in this paper.

Electrostatic nonlinear plasma theory has been developing for more than half a century (Sagdeev and Galeev, 1969; Davidson, 1972). Important issues, such as the excitation, propagation, and effects of nonlinear waves, have been extensively studied since the 1950s when Bernstein, Green, and Kruskal (BGK) (1957) predicted the first non-wave, 1D solitary phase-space structures (see, e.g., Infeld and Rowlands 2000). Up to now, numerous studies have been carried out since the 1970s, with an extension to 2-D and 3-D approaches (see a recent review by Eliasson and Shukla 2006). For instance, Shukla and Yu (1978) and Yu et al. (1980) gave exact stationary solutions for the solitons propagating obliquely in a two-component (electron and ion), low- $\beta$ plasma $\left(T_{\mathrm{e}} \gg T_{\mathrm{i}}\right)$ in a constant magnetic field; and, observationally, Temerin et al. (1979) found three shapes of electrostatic solitary structures (namely, sinusoidal, sawtooth, and spicky/bipolar) from the S3-3 data. In a generalized study, Lee and Kan (1981) obtained nonlinear IA and IC waves, and IA solitons, followed by Nakamura and Sugai (1996)'s study on a three-component (warm ion, cold and energetic electrons) system with a pseudo-potential method (i.e., the
"Sagdeev potential"). In such a system, Chatterjee and Roychoudhury (1997) found that, when the ion temperature increases, the amplitude of the IA solitary waves increases while the self-similar coordinate (denoted by $\xi=x-V t$ ) decreases. Here, $x$ is the 1-D coordinate, $V$ the phase speed of solitons, and $t$ the time. For a system containing cold multiionic components, Das et al. (2000) claimed that a power expansion technique could lead to higher order nonlinear IA wave equations which could yield various solitary wave propagations, such as, spiky solitons, collapsed or explosive ones, and double layers. Pottelette et al. (2003) studied the excitation of IA solitons in a system composed of a cool ion component and two thermal electron populations. Recently, Ma and Hirose (2009) performed a parameterized study on propagating IA solitary waves in a system which consisted of a warm ion component and two-temperature isothermal electrons (background electrons and energetic ones). These studies adopted a charge-neutrality condition and no HF ingredients appear to superimpose upon the simple LF wave packets.

Contrary to the single concept, "oscillitons", appeared in the early 2000s (Sauer et al., 2001) to describe nonlinear, multi-frequency, electromagnetic structures, the singlefrequency, electrostatic envelopes were designated in literature by many nomenclatural terms. Besides well-known theoretical descriptions like "BGK (solitary) waves", "solitary (electrostatic) waves", "phase-space (electron or ion) holes", Dupree (1972) described them as "clumps", while Chiueh and Diamond (1986) called them "density granulations" or "space-charge holes" (either over-densed or under-densed). Observationally, satellite projects verified these structures via spikelet electric field measurements at numerous sites in geospace, such as, OVO-1 in auroral regions (Heppner, 1969), IMP-7 in the neutral sheet (Scarf et al., 1974), Hawkeye 1 \& IMP 6 near the magnetic equator (Gurnett, 1976), Hawkeye 1 \& IMP 8 in the outer radiation zone (Gurnett and Frank, 1976), IMP-8 in the plasma sheet boundary layer (Gurnett et al., 1976), DE-1 at the polar magnetosphere (Gurnett et al., 1983) and the dayside magnetopause (LaBelle et al., 1987), Viking in auroral zones (Pottelette et al., 1988; Hultqvist, 1989) and the entire cleft region (Dubouloz et al., 1991), ISEE (Cattell et al., 1986), AMPTE (Baumjohann et al., 1989), and GEOTAIL (Matsumoto et al., 1994; Kojima et al., 1997) in the magnetotail; Wind/WAVES in the dusk magnetopause (Farrell et al., 2002), FAST in the auroral downward current region (see Table 2 in Ergun et al., 2003), and, Cluster in the vicinity of the polar cusp (Lin et al., 2006). They were named "non-wave cavitons", "broadband electrostatic noises (BENs)", "(non-wave) solitons", "electrostatic holes", "kinks", "electrostatic shocks", "(weak or strong) double layers", "bipolar/tripolar electric-field structures", etc. See the Introduction section of Ma and Hirose (2009) for details. A common feature of data in these measurements lies in a single-frequency spectrum at either ioncyclotron (IC) or ion-acoustic (IA) frequencies. 
By employing Verheest et al. (2004)'s approach, we pay attention to parallel-propagating, nonlinear solitary waves to see the impact of the charge non-neutrality condition on the excitation and the features of the multi-frequency structures, called "electrostatic oscillitons". In order to provide a direct comparison between the results under the present charge non-neutrality condition and what was introduced under the charge neutrality condition (Ma, 2010), we chose a cylindrical geometry. In fact, cylindrically symmetric systems are ubiquitously observed in geo-space by a multitude of measurements from, e.g., ground-based imagers (Pimenta et al., 2001), rockets (Earle et al., 1989; Moore et al., 1996), and satellites (Pickett et al., 2004; Vaivads et al., 2004; De Keyser et al., 2005).

In the cylindrical frame, where $\boldsymbol{B}$ is along an axial $\mathrm{z}$ direction, Ma (2010) used a self-similar, two-fluid plasma model under a charge neutrality condition to show the features of the parallel-propagating, simple nonlinear IA waves, from which only single-frequency waves can evolve. Numerical calculations exposed that, similar to the Cartesian case, the three types of nonlinear structures mentioned above (sinusoidal, sawtooth, and spiky or bipolar) still exist, whereas Cluster satellite data (Cattell et al., 2003) can be explained by simulated diverging and converging electric shock packets. By contrast, the initiative of the present paper is to answer a fundamental question: Can nonlinear multi-frequency "oscillitons" be excited electrostatically by the charge nonneutrality? The work is layed out as follows: Section 2 sets up a generalized self-similar, two-fluid model in a cylindrical geometry for a two-component electron-ion plasma system. Section 3 introduces basic features of IA solitary waves in regions satisfying the charge-neutrality condition via a numerical approach. Section 4 relaxes the condition to charge non-neutrality and provides a parameterized study to show the modulation of solitary structures to oscillitons. The influences of input parameters and initial conditions on oscillitons are also described. Section 5 presents a summary and a discussion. An appendix describes briefly the linear modes excited in a cylindrical system.

\section{Two-fluid model}

In order to provide the most basic picture for the modulation of charge non-neutrality on the emergence and propagation of electrostatic IA solitary oscillitons, and thus to gain important insights into more complicated situations while still being able to illustrate the process clearly, we focus on regions rockets (e.g., Geodesic) and satellites (e.g., Freja, FAST, and Cluster) have visited frequently, such as the ionospheric auroral F-layer, acceleration region, bow shock, and magnetopause. In these regions, the plasma $\beta$ is much less than 1 and thus the waves triggered are electrostatic; however, the ion magnetic gyrofrequency $\left(\Omega_{\mathrm{i}}\right)$ is not always much smaller than the ion plasma frequency $\left(\omega_{\mathrm{pi}}\right)$ but the ratio between them varies greatly from one region to the other.

We employed a two-fluid model in a cylindrical geometry $(r, \phi, z)$ with $\boldsymbol{B}=B \hat{\boldsymbol{e}}_{\mathrm{Z}}$ (where $B$ is constant, $\hat{\boldsymbol{e}}_{\mathrm{Z}}$ is the unit vector along z-axis). The model takes into account isothermal electron and adiabatic ion fluids, with $v_{\mathrm{Te}} \gg v_{\mathrm{Ti}}$, where $v_{\mathrm{Te}}=\sqrt{2 k_{\mathrm{B}} T_{\mathrm{e}} / m_{\mathrm{e}}}$ and $v_{\mathrm{Ti}}=\sqrt{2 k_{\mathrm{B}} T_{\mathrm{i} 0} / m_{\mathrm{i}}}$ are the electron and ion thermal speeds, respectively, in which $k_{\mathrm{B}}$ is Boltzmann's constant, $T_{\mathrm{e}}$ and $T_{\mathrm{i} 0}$ are the electron temperature and ion initial temperature, respectively, and $m_{\mathrm{e}}$ and $m_{\mathrm{i}}$ are their masses, respectively.

\subsection{Equations of electron and ion fluids}

Up to the present, few studies have been performed on the excitation of nonlinear "IA oscillitons" in a two-fluid plasma system. We thus exclude the magnetic filed variation (i.e., under an electrostatic condition which is in agreement with the low $\beta$ condition), and neglect the electron inertia due to $m_{\mathrm{e}} \ll m_{\mathrm{i}}$, in the present article, so as to minimize external distractions from the focus on the effect of the charge nonneutrality condition. It should be mentioned here that, in the case including variation and inertia, Ma and Hirose (2010) showed that electromagnetic "lower-hybrid oscillitons" are triggered.

For the electron fluid considered throughout this paper, both the momentum equation and the isothermal equation of state provide

$N_{\mathrm{e}}=e^{\Phi}$

where $N_{\mathrm{e}}=n_{\mathrm{e}} / n_{0}$ is the dimensionless electron density in which $n_{\mathrm{e}}$ and $n_{0}$ are the dimensional electron density and the uniform background plasma density, respectively, and $\Phi=$ $e \varphi /\left(k_{\mathrm{B}} T_{\mathrm{e}}\right)$ in which $\varphi$ is the electrostatic potential satisfying $\boldsymbol{E}=-\nabla \varphi$ where $\boldsymbol{E}$ denotes the electrostatic electric field. For ions, by using $n_{\mathrm{i}},\left\{u_{r}, u_{\phi}, u_{\mathrm{z}}\right\}$, and $t$ to denote ion density, velocity components, and time, respectively, we write the ion fluid equations as follows:

1. Continuity equation

$$
\frac{\partial n_{\mathrm{i}}}{\partial t}+\frac{\partial\left(n_{\mathrm{i}} u_{r}\right)}{\partial r}+\frac{\partial\left(n_{\mathrm{i}} u_{\mathrm{z}}\right)}{\partial \mathrm{z}}=-\frac{n_{\mathrm{i}} u_{r}}{r}
$$

2. Radial momentum equation

$$
\begin{aligned}
\frac{\partial u_{r}}{\partial t}+ & u_{r} \frac{\partial u_{r}}{\partial r}+u_{\mathrm{z}} \frac{\partial u_{r}}{\partial \mathrm{z}}= \\
& =\frac{u_{\phi}^{2}}{r}-v_{\mathrm{Ti}}^{2} \frac{\partial}{\partial r}\left(\frac{n_{\mathrm{i}}}{n_{\mathrm{i} 0}}\right)-\frac{\Omega_{\mathrm{i}}}{B} \frac{\partial \varphi}{\partial r}+\Omega_{\mathrm{i}} u_{\phi}
\end{aligned}
$$

3. Azimuthal momentum equation

$$
\frac{\partial u_{\phi}}{\partial t}+u_{r} \frac{\partial u_{\phi}}{\partial r}+u_{\mathrm{z}} \frac{\partial u_{\phi}}{\partial \mathrm{z}}=-\frac{u_{r} u_{\phi}}{r}-\Omega_{\mathrm{i}} u_{r}
$$


4. Axial momentum equation

$$
\frac{\partial u_{\mathrm{z}}}{\partial t}+u_{r} \frac{\partial u_{\mathrm{z}}}{\partial r}+u_{\mathrm{z}} \frac{\partial u_{\mathrm{z}}}{\partial \mathrm{z}}=-v_{\mathrm{Ti}}^{2} \frac{\partial}{\partial \mathrm{z}}\left(\frac{n_{\mathrm{i}}}{n_{\mathrm{i} 0}}\right)-\frac{\Omega_{\mathrm{i}}}{B} \frac{\partial \varphi}{\partial \mathrm{z}}
$$

\section{Poisson's equation}

$$
\frac{1}{r} \frac{\partial}{\partial r}\left(r \frac{\partial \varphi}{\partial r}\right)+\frac{\partial^{2} \varphi}{\partial \mathrm{z}^{2}}=\frac{e\left(n_{\mathrm{e}}-n_{\mathrm{i}}\right)}{\epsilon_{0}}
$$

where $\Omega_{\mathrm{i}}=e B / m_{\mathrm{i}}$, and $\epsilon_{0}$ is the vacuum permittivity.

It can be noticed that all derivative terms along the $\phi$ direction do not occur due to the symmetric nature of the cylindrical system. Besides, because the adiabatic index is 2, we have applied $p_{\mathrm{i}} n_{\mathrm{i}}^{-2}=$ Const., as well as the equation of state, $p_{\mathrm{i} 0}=n_{0} k_{\mathrm{B}} T_{\mathrm{i} 0}$, in deriving the momentum equations.

By normalizing all variables with $N_{\mathrm{i}}=n_{\mathrm{i}} / n_{0}, R=r / \rho_{\mathrm{i}}$ (where $\rho_{\mathrm{i}}=c_{\mathrm{s}} / \Omega_{\mathrm{i}}$ is the ion gyro-radius in which $c_{\mathrm{s}}=$ $\sqrt{k_{\mathrm{B}} T_{\mathrm{e}} / m_{\mathrm{i}}}$ is the ion acoustic speed), $Z=\mathrm{z} / \rho_{\mathrm{i}}, V_{r}=u_{r} / c_{\mathrm{s}}$, $V_{\phi}=u_{\phi} / c_{\mathrm{s}}, V_{\mathrm{z}}=u_{\mathrm{z}} / c_{\mathrm{s}}, \tau=\Omega_{\mathrm{i}} t$, we obtain a dimensionless set of above differential equations as follows:

$$
\begin{aligned}
& \frac{\partial N_{\mathrm{i}}}{\partial \tau}+\frac{\partial\left(N_{\mathrm{i}} U_{r}\right)}{\partial R}+\frac{\partial\left(N_{\mathrm{i}} U_{\mathrm{z}}\right)}{\partial Z}=-\frac{N_{\mathrm{i}} U_{r}}{R} \\
& \frac{\partial U_{r}}{\partial \tau}+U_{r} \frac{\partial U_{r}}{\partial R}+U_{\mathrm{z}} \frac{\partial U_{r}}{\partial Z}=\frac{U_{\phi}^{2}}{R}-\frac{\partial N_{\mathrm{i}}}{\partial R}-\frac{\partial \Phi}{\partial R}+U_{\phi} \\
& \frac{\partial U_{\phi}}{\partial \tau}+U_{r} \frac{\partial U_{\phi}}{\partial R}+U_{\mathrm{z}} \frac{\partial U_{\phi}}{\partial Z}=-\frac{U_{r} U_{\phi}}{R}-U_{r} \\
& \frac{\partial U_{\mathrm{z}}}{\partial \tau}+U_{r} \frac{\partial U_{\mathrm{z}}}{\partial R}+U_{\mathrm{z}} \frac{\partial U_{\mathrm{z}}}{\partial Z}=-\frac{1}{\xi_{T}} \frac{\partial N_{\mathrm{i}}}{\partial Z}-\frac{\partial \Phi}{\partial Z} \\
& \frac{1}{R} \frac{\partial}{\partial R}\left(R \frac{\partial \Phi}{\partial R}\right)+\frac{\partial^{2} \Phi}{\partial Z^{2}}=\xi_{\omega}^{2}\left(N_{\mathrm{e}}-N_{\mathrm{i}}\right)
\end{aligned}
$$

in which $\xi_{T}=c_{\mathrm{s}}^{2} / v_{\mathrm{Ti}}^{2}=T_{\mathrm{e}} /\left(2 T_{\mathrm{i} 0}\right)$ and $\xi_{\omega}=\omega_{\mathrm{pi}} / \Omega_{\mathrm{i}}=\rho_{\mathrm{i}} / \lambda_{\mathrm{De}}$ where $\omega_{\mathrm{pi}}$ is the ion plasma frequency, and $\lambda_{\mathrm{De}}=c_{\mathrm{s}} / \omega_{\mathrm{pi}}=$ $\sqrt{\epsilon_{0} k_{\mathrm{B}} T_{\mathrm{e}} /\left(n_{0} e^{2}\right)}$ is the electron Debye length. Naturally, $c_{\mathrm{s}} k_{\mathrm{De}}=\omega_{\mathrm{pi}}$ where $k_{\mathrm{De}}=\lambda_{\mathrm{De}}^{-1}$ is the electron Debye wave number.

In such a system, linear IA and IC waves can be excited. By linearizing Eqs. (7)-(11), we obtain various linear wave modes as given in Appendix A.

\subsection{Generalized nonlinear self-similar equations}

Superimposing upon these background linear oscillations, there exist nonlinear solitary waves the features of which can be obtained by solving a set of self-similar equations of Eqs. (7)-(11) via introducing a self-similar parameter $X$ (Lee and Kan, 1981; Shi et al., 2001):

$X=\frac{\alpha_{1}}{M} R+\frac{\alpha_{2}}{M} Z-\tau$ where $M$ is the Mach number, $\alpha_{1}=\sin \theta$, and $\alpha_{2}=\cos \theta$ in which $\theta$ is the inclination angle between the propagation direction and the magnetic field. Using this self-similar transformation, we have

$$
\frac{\partial}{\partial \tau}=-\frac{\mathrm{d}}{\mathrm{d} X}, \frac{\partial}{\partial R}=\frac{\alpha_{1}}{M} \frac{\mathrm{d}}{\mathrm{d} X}, \frac{\partial}{\partial Z}=\frac{\alpha_{2}}{M} \frac{\mathrm{d}}{\mathrm{d} X}
$$

Equations (7)-(11) become

$$
\begin{gathered}
\left(1-\frac{\alpha_{1} U_{r}+\alpha_{2} U_{\mathrm{z}}}{M}\right) \frac{\mathrm{d} \ln N_{\mathrm{i}}}{\mathrm{d} X}- \\
-\frac{1}{M} \frac{\mathrm{d}\left(\alpha_{1} U_{r}+\alpha_{2} U_{\mathrm{z}}\right)}{\mathrm{d} X}=\frac{U_{r}}{R} \\
\left(1-\frac{\alpha_{1} U_{r}+\alpha_{2} U_{\mathrm{z}}}{M}\right) \frac{\mathrm{d} U_{r}}{\mathrm{~d} X}= \\
=-\left(\frac{U_{\phi}}{R}+1\right) U_{\phi}+\frac{\alpha_{1}}{M} \frac{\mathrm{d}\left(N_{\mathrm{i}}+\Phi\right)}{\mathrm{d} X} \\
\left(1-\frac{\alpha_{1} U_{r}+\alpha_{2} U_{\mathrm{z}}}{M}\right) \frac{\mathrm{d} U_{\phi}}{\mathrm{d} X}=\left(\frac{U_{\phi}}{R}+1\right) U_{r} \\
\left(1-\frac{\alpha_{1} U_{r}+\alpha_{2} U_{\mathrm{z}}}{M}\right) \frac{\mathrm{d} U_{\mathrm{z}}}{\mathrm{d} X}=\frac{\alpha_{2}}{M} \frac{\mathrm{d}\left(\frac{N_{\mathrm{i}}}{\xi_{T}}+\Phi\right)}{\mathrm{d} X} \\
\frac{\mathrm{d}^{2} \Phi}{\mathrm{d} X^{2}}+\alpha_{1}^{2} \frac{\mathrm{d} \ln R}{\mathrm{~d} X} \frac{\mathrm{d} \Phi}{\mathrm{d} X}=M^{2} \xi_{\omega}^{2}\left(N_{\mathrm{e}}-N_{\mathrm{i}}\right)
\end{gathered}
$$

We have concentrated on parallel-propagating solitary waves in this article, i.e., $\theta=0^{\circ}$. Thus, $\partial / \partial R=0$, meaning all variables are independent of $R$. Note that $R$ occurs in equations as a constant to represent the radius of curvature of the flow streamline intersecting the magnetic field lines on which the equation is going to be solved. It provides the centrifugal and Coriolis forces absent in rectilinear flows and behaves as an input parameter of the system. In this case, the above set of equations takes a much simpler form as follows:

$$
\begin{aligned}
& \left(1-\frac{U_{\mathrm{z}}}{M}\right) \frac{\mathrm{d} \ln N_{\mathrm{i}}}{\mathrm{d} X}-\frac{1}{M} \frac{\mathrm{d} U_{\mathrm{z}}}{\mathrm{d} X}=\frac{U_{r}}{R} \\
& \left(1-\frac{U_{\mathrm{z}}}{M}\right) \frac{\mathrm{d} U_{r}}{\mathrm{~d} X}=-\left(\frac{U_{\phi}}{R}+1\right) U_{\phi} \\
& \left(1-\frac{U_{\mathrm{z}}}{M}\right) \frac{\mathrm{d} U_{\phi}}{\mathrm{d} X}=\left(\frac{U_{\phi}}{R}+1\right) U_{r} \\
& \left(1-\frac{U_{\mathrm{z}}}{M}\right) \frac{\mathrm{d} U_{\mathrm{z}}}{\mathrm{d} X}=\frac{1}{M} \frac{\mathrm{d}\left(\frac{N_{\mathrm{i}}}{\xi_{T}}+\Phi\right)}{\mathrm{d} X} \\
& \frac{\mathrm{d}^{2} \Phi}{\mathrm{d} X^{2}}=M^{2} \xi_{\omega}^{2}\left(N_{\mathrm{e}}-N_{\mathrm{i}}\right)
\end{aligned}
$$




\section{Features of solitary waves under charge-neutrality conditions}

As shown in Table 1, the condition of $\rho_{\mathrm{i}} \gg \lambda_{\mathrm{De}}$ (or, equivalently, $\left.\Omega_{\mathrm{i}} \ll \omega_{\mathrm{pi}}\right)$ is valid at lower altitudes, e.g., the ionospheric F-layer. Thus, the charge separation effects can be satisfactorily neglected, and the charge quasi-neutrality condition (namely, $N_{\mathrm{i}} \approx N_{\mathrm{e}}$ ) is met. By using $N_{\mathrm{i}} \approx N_{\mathrm{e}}$ and $N_{\mathrm{e}}=e^{\Phi}$ in Eqs. (19)-(23), we obtain

$$
\begin{aligned}
& \frac{\mathrm{d} V_{r}}{\mathrm{~d} X}-\frac{V_{\mathrm{z}}}{M} \frac{\mathrm{d} V_{r}}{\mathrm{~d} X}=-\frac{V_{\phi}^{2}}{R}-V_{\phi} \\
& \frac{\mathrm{d} V_{\phi}}{\mathrm{d} X}-\frac{V_{\mathrm{z}}}{M} \frac{\mathrm{d} V_{\phi}}{\mathrm{d} X}=\frac{V_{r} V_{\phi}}{R}+V_{r} \\
& \frac{\mathrm{d} V_{\mathrm{z}}}{\mathrm{d} X}-\frac{V_{\mathrm{z}}}{M} \frac{\mathrm{d} V_{\mathrm{z}}}{\mathrm{d} X}=\frac{\zeta}{M} \frac{\mathrm{d} \Phi}{\mathrm{d} X} \\
& \left(1-\frac{V_{\mathrm{z}}}{M}\right) \frac{\mathrm{d} \Phi}{\mathrm{d} X}-\frac{1}{M} \frac{\mathrm{d} V_{\mathrm{z}}}{\mathrm{d} X}=\frac{V_{r}}{R}
\end{aligned}
$$

in which $\zeta=1+\xi_{T}^{-1}$. Considering $\Phi_{0}=0$ at $X=0$, the third equation gives

$V_{\mathrm{z}}=M(1 \pm \sqrt{1-\Psi})$

where $\Psi=2\left(\zeta \Phi / M^{2}+V_{\mathrm{z} 0} / M\right)-V_{\mathrm{z} 0}^{2} / M^{2}$. Clearly, the dual solutions of $V_{\mathrm{z}}$ means that there are two branches of propagating solitary waves with $V_{\mathrm{z} 0}=0$ and $2 M$, respectively.

\subsection{Forward-propagating solitary waves}

A branch of the solitary waves has $V_{\mathrm{z}}=M(1+\sqrt{1-\Psi}) \geq$ $M$. Because $V_{\mathrm{z}} \geq 0$, it propagates forward along magnetic field lines. Applying the $V_{\mathrm{Z}}$ expression in Eqs. (24)-(27) produce

$\left\{\begin{array}{l}\sqrt{1-\Psi} \frac{\mathrm{d} V_{r}}{\mathrm{~d} X}=\left(\frac{V_{\phi}}{R}+1\right) V_{\phi} \\ \sqrt{1-\Psi} \frac{\mathrm{d} V_{\phi}}{\mathrm{d} X}=-\left(\frac{V_{\phi}}{R}+1\right) V_{r} \\ \left(\frac{M^{2}}{\zeta} \sqrt{1-\Psi}-\frac{1}{\sqrt{1-\Psi}}\right) \frac{\mathrm{d} \Psi}{\mathrm{d} X}=-2 \frac{V_{r}}{R}\end{array}\right.$

We use $N_{\mathrm{i}} \approx e^{\Phi}$, and introduce a dimensionless electric field $E_{\mathrm{i}}$ determined by

$E_{\mathrm{i}}=-\frac{1}{M} \frac{\mathrm{d} \Phi}{\mathrm{d} X}=-\frac{M}{2 \zeta} \frac{\mathrm{d} \Psi}{\mathrm{d} X}$

in a unit of $E_{0}=c_{\mathrm{s}} B$. After taking $T_{\mathrm{e}}=10 T_{\mathrm{i} 0}$ which gives $\xi_{T}=5$ and $\zeta=1.2$, and using input parameters $M=1.8$ and $R=0.9$, we calculate Eq. (29) and expose features of this forward-propagating branch in Fig. 1. The axial speed $V_{\mathrm{z}}$, ion density $N_{\mathrm{i}}$, and wave-field strength $E_{\mathrm{i}}$ are shown in the three top-row, middle-row, and bottom-row panels, respectively, while the three left-column, middle-column, and right-column panels display solitons with a sinusoidal shape in $E_{\mathrm{i}}$ excited by a slow-speed azimuthal flow of $V_{\phi 0}=0.1$, that with a sawtooth shape in $E_{\mathrm{i}}$ excited by a medium-speed azimuthal flow of $V_{\phi 0}=0.6$, and that with a bipolar shape in $E_{\mathrm{i}}$ excited by a fast-speed azimuthal flow of $V_{\phi 0}=0.89$, respectively. Other boundary conditions are $\left\{V_{r}, \Psi\right\}=\{0,0\}$.

The sinusoidal, sawtooth and bipolar (or spiky) shapes in $E_{\mathrm{i}}$ are the three well-known structures observed in space. Corresponding to the three $E_{\mathrm{i}}$ structures, both $V_{\mathrm{z}}$ and $N_{\mathrm{i}}$ have oscillations of the same frequency. Strikingly, for a phase speed $M=1.8$, the amplitude of solitons in $V_{\mathrm{z}}$ enhances from the initial value of 3.6, higher than $M$, to 3.8 in the sinusoidal case, 4.6 in the sawtooth case, and 5.9 in the bipolar case, respectively, representing even higher speeds than $M$; by contrast, $N_{\mathrm{i}}$ decreases from the initial value of 1 to 0.7 , $0.1,0.002$, respectively, in the three cases. It is clear that (1) solitons exist in boundaries of density holes, or, equivalently, they are spatial and temporal islands, the density of which is higher than the surroundings; (2) soliton ions have a slower speed in propagation but constitute violently changing electric field strengths; (3) solitary structures are modulated by $V_{\phi 0}$ in that a small-amplitude, sinusoidal mode evolves to a medium-amplitude, sawtooth mode, and finally a highamplitude, bipolar mode when $V_{\phi 0}$ increases; (4) the wavelengths $X_{\omega}$ of the periodic structures are correlated with the amplitudes: they are $X_{\omega}=6.7,11.4,85$ (in units of $\rho_{\mathrm{i}}$ ), respectively, corresponding to the three levels of amplitudes.

In the last case, in particular, diverging shocks (Ergun et al., 1998b) are found undoubtedly to have developed from the middle case. They are featured by a negative electric field followed by a positive one. In addition, calculations with a changing $M$ under $M>1$ expose that $N_{\mathrm{i}}$ is never larger than 1 , but moves to a minimum which is smaller at a faster drift as already presented in the figure. Furthermore, $V_{\phi 0}$ cannot be larger than 1 . This means that the azimuthal speed is unable to surpass the local acoustic speed for soliton solutions to be available.

\subsection{Backward-propagating solitary waves}

The other branch of the solitary waves has $V_{\mathrm{z}}=M(1-$ $\sqrt{1-\Psi}$ ). Applying the $V_{\mathrm{Z}}$ expression in Eqs. (24)-(27) produces

$\left\{\begin{array}{l}\sqrt{1-\Psi} \frac{\mathrm{d} V_{r}}{\mathrm{~d} X}=-\left(\frac{V_{\phi}}{R}+1\right) V_{\phi} \\ \sqrt{1-\Psi} \frac{\mathrm{d} V_{\phi}}{\mathrm{d} X}=\left(\frac{V_{\phi}}{R}+1\right) V_{r} \\ \left(\frac{M^{2}}{\zeta} \sqrt{1-\Psi}-\frac{1}{\sqrt{1-\Psi}}\right) \frac{\mathrm{d} \Psi}{\mathrm{d} X}=2 \frac{V_{r}}{R}\end{array}\right.$

By comparison with the forward-propagating branch, we find that the density structures reproduce the previous cases totally, as exhibited by Fig. 2 under same conditions. However, there are three different features: (1) $V_{\mathrm{Z}}$ is always smaller than zero, meaning that this is a backward-propagating mode; (2) the amplitude of $V_{\mathrm{z}}$ is always smaller than that of the forward-propagating mode, indicating that the propagation speed is slower; (3) the solitons are converging shocks characterized by a positive electric field followed by a negative one (McFadden et al. 2003). 

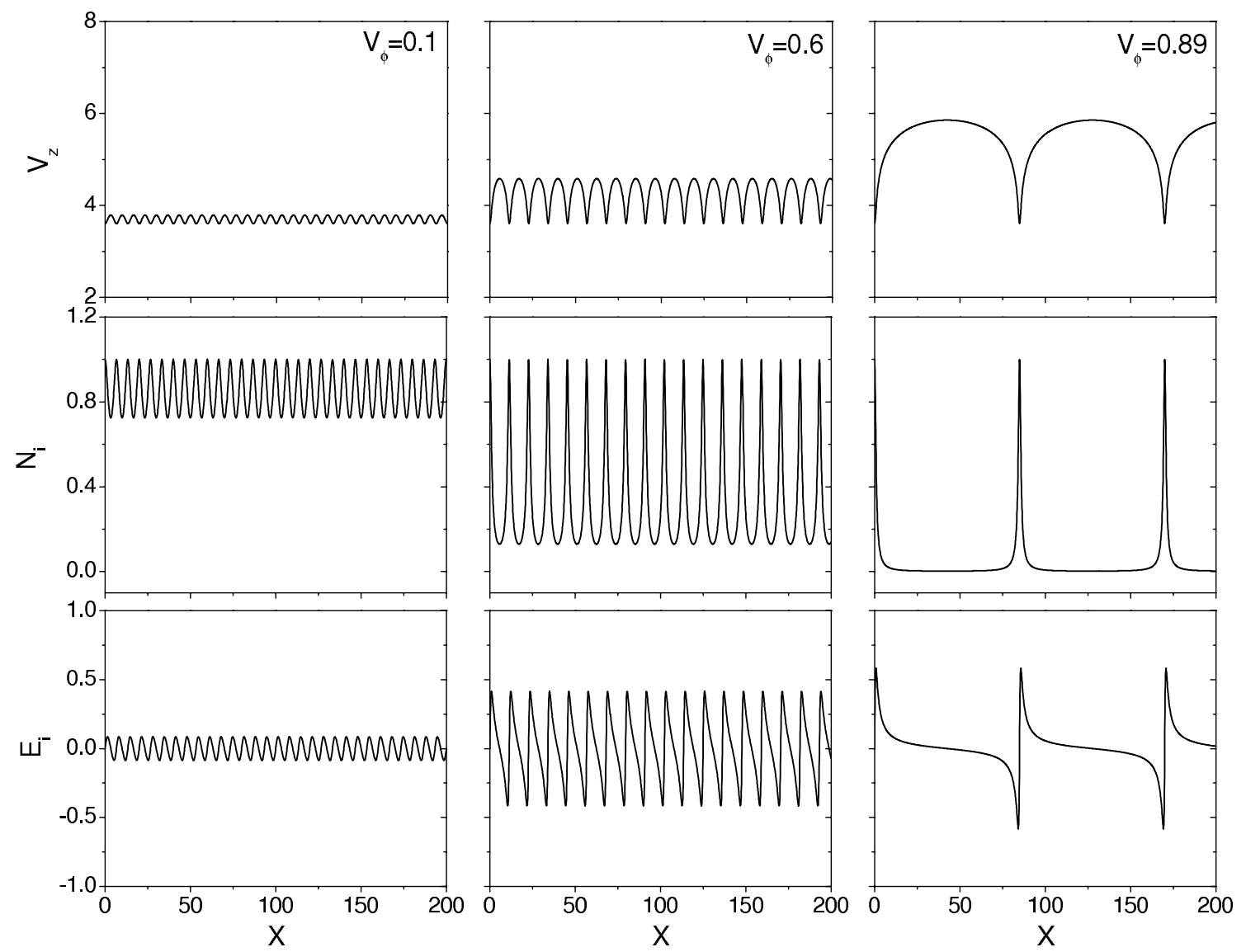

Fig. 1. Features of the forward-propagating branch of solitary waves via the axial speed $V_{\mathrm{Z}}$ (top-row panels), ion density $N_{\mathrm{i}}$ (middle-row panels), and wave-field strength $E_{\mathrm{i}}$ (bottom-row panels): (1) solitons with a sinusoidal shape in $E_{\mathrm{i}}$ excited by a slow-speed azimuthal flow of $V_{\phi 0}=0.1$ (left-column panels); (2) solitons with a sawtooth shape in $E_{\mathrm{i}}$ excited by a medium-speed azimuthal flow of $V_{\phi 0}=0.6$ (middlecolumn panels); (3) solitons with a bipolar shape in $E_{\mathrm{i}}$ excited by a fast-speed azimuthal flow of $V_{\phi 0}=0.89$ (right-column panels). Input parameters: $\zeta=1.2, M=1.8$, and $R=0.9$; Boundary conditions: $\left\{V_{r}, \Psi\right\}=\{0,0\}$.

\subsection{FFT spectra of $E_{i}$}

Equations (24)-(27) describe localized, coherent solitary waves which are excited in the two-fluid system by the balance of nonlinearity and the dispersive effect (Davidson, 1972; Drazin, 1984, and references therein). It can be noted that it is suitable for nonlinear solitary waves driven in regions where the charge quasi-neutrality is satisfied to superimpose upon background linear waves the dispersive characteristics of which can be seen from the propagation features of nonlinear structures.

In Eqs. (24)-(27), all spatial parameters are normalized by $\rho_{\mathrm{i}}$. Thus, $\xi_{\omega}^{-1}$ (or, $\Omega_{\mathrm{i}} / \omega_{\mathrm{pi}}$ ) is as a matter of fact the exact expression of the dimensionless $\lambda_{\mathrm{De}}$. Obviously, it is smaller than 1 as observed data from 0.0009 to 0.25 shown in Table 1. Undoubtedly, $\lambda_{\mathrm{De}} \ll X_{\omega}$, or $k_{\mathrm{De}} \gg k_{X}=X_{\omega}^{-1}$. As a result, we can readily use the dimension-free expression $\omega^{2}=\zeta k_{X}^{2}$ (i.e., Eq. A9), to evaluate the dispersive IA signatures which is the only mode existing in the cases discussed above. Easily, we have

$$
\omega=\frac{\sqrt{\zeta}}{X_{\omega}}
$$

which provides $\omega_{X}=0.163,0.096,0.013$ in accordance with the three $X_{\omega}$ values. These should constitute the three dominant oscillation frequencies of the IA mode, accompanied by a series of harmonics due to the fact that the solitary waveforms cannot be described by pure sine functions, if we analyze the periodic $E_{\mathrm{i}}$ structures of the solitary waves in a frequency regime.

Figure 3 demonstrate such an analysis via the FFT algorithm. The upper three panels depict the spectra of the forward-propagating $E_{\mathrm{i}}$ structures, while the lower three panels reveal that of the backward-propagating ones: the two left-column panels are for the sinusoidal solitons with $V_{\phi 0}=0.1$; the two middle-column panels are for the sawtooth solitons with $V_{\phi 0}=0.6$; and the two right-column panels are the bipolar solitons with $V_{\phi 0}=0.89$. The panels reveal the three main peaks at $0.160,0.090$, and 0.012 , respectively, for the three $E_{\mathrm{i}}$ structures, and confirm the harmonics with $0.300,0.450,0.600, \cdots$, in the sinusoidal case; 0.175 , 

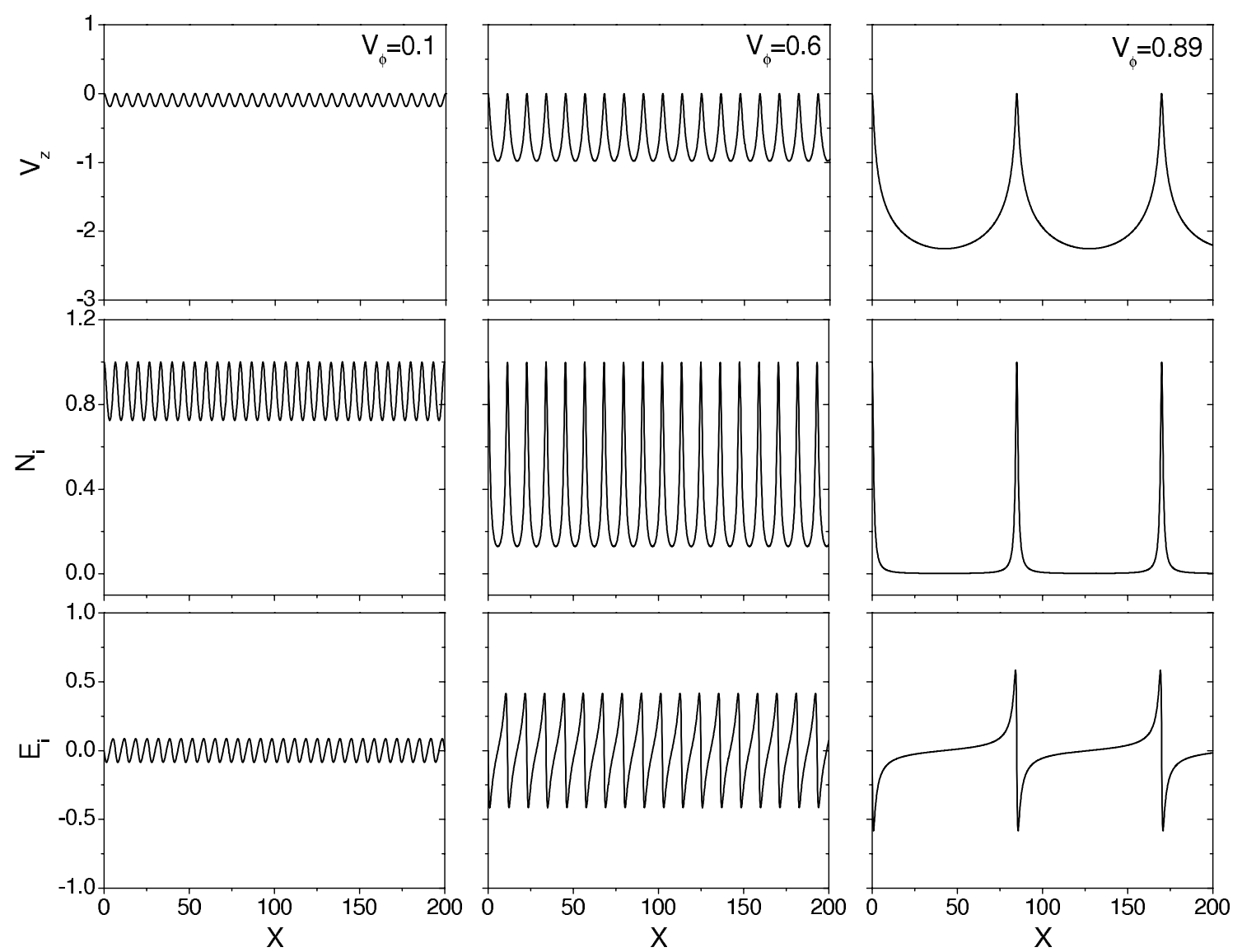

Fig. 2. Same as Fig. 1 but that of the backward-propagating branch of solitary-waves. Note that the density structures are totally the same as those in the previous figure.

$0.265,0.350, \cdots$, in the sawtooth case; and $0.023,0.035$, $0.047, \cdots$, in the bipolar case, respectively.

Tiny errors between $\omega_{X}$ predicted theoretically by using Eq. (32) and the FFT data can be noticed here. By adjusting the simulation scales in $X$, we know that the longer the lengths in $X$, the closer the FFT frequencies to the $\omega_{X}$ values. Thus, we realize that the difference is contributed by the limited length in practical calculations. We would also like to point out that (1) both forward and backward propagating solitary waves contribute to an identical frequency spectrum, not only in power amplitudes, but also in frequency shifts of the noise bands; (2) all the three $E_{\mathrm{i}}$ structures offer IA frequency-sweeping bands smaller than the IC frequency, $\Omega_{\mathrm{i}}$, although the bipolar noises can extend a little above them. (3) Any extra factors considered to break up the plasma neutrality in the two-fluid system will introduce new oscillating modes superimposing upon the IA mode, however, disappearing increasingly with the strengthening of the charge-neutrality condition, as will be seen below.

\section{Features of solitary waves under charge non-neutrality conditions}

The non-neutrality of charges means that $\rho_{\mathrm{i}} \gg \lambda_{\text {De }}$ is not satisfied anymore. In this case, the charge neutrality condition, $N_{\mathrm{i}} \approx N_{\mathrm{e}}$, does not maintain in the two-fluid system. Space charges $N_{\mathrm{sc}}=N_{\mathrm{i}}-N_{\mathrm{e}}$ are brought into into play in Eqs. (19)-(23), while electrons continues to keep $N_{\mathrm{e}}=e^{\Phi}$ as an isothermal fluid. For the ion fluid, by substituting $M-U_{z}$ with $U_{z}$, we obtain a new set of equations from Eqs. (19)(23) as follows:

$$
\left.\begin{array}{l}
\frac{\mathrm{d} N_{\mathrm{i}}}{\mathrm{d} X}=M N_{\mathrm{i}} \frac{\frac{U_{r} U_{\mathrm{z}}}{R}-E}{U_{\mathrm{z}}^{2}-\frac{N_{\mathrm{i}}}{\xi_{T}}} \\
\frac{\mathrm{d} U_{r}}{\mathrm{~d} X}=-M \frac{U_{\phi}}{U_{\mathrm{z}}}\left(\frac{U_{\phi}}{R}+1\right) \\
\frac{\mathrm{d} U_{\phi}}{\mathrm{d} X}=M \frac{U_{r}}{U_{\mathrm{z}}}\left(\frac{U_{\phi}}{R}+1\right) \\
\frac{\mathrm{d} U_{\mathrm{z}}}{\mathrm{d} X}=M \frac{U_{\mathrm{z}} E-\frac{N_{\mathrm{i}} U_{r}}{R \xi_{T}}}{U_{\mathrm{z}}^{2}-\frac{N_{\mathrm{i}}}{\xi_{T}}} \\
\frac{\mathrm{d} \Phi}{\mathrm{d} X}=-M E \\
\frac{\mathrm{d} E}{\mathrm{~d} X}=M \xi_{\omega}^{2} N_{\mathrm{sc}}
\end{array}\right\}
$$

An obvious difference of this set of equations from Eqs. (24)-(27) lies in that $V_{\mathrm{z}}$ is unable to be solved as a 

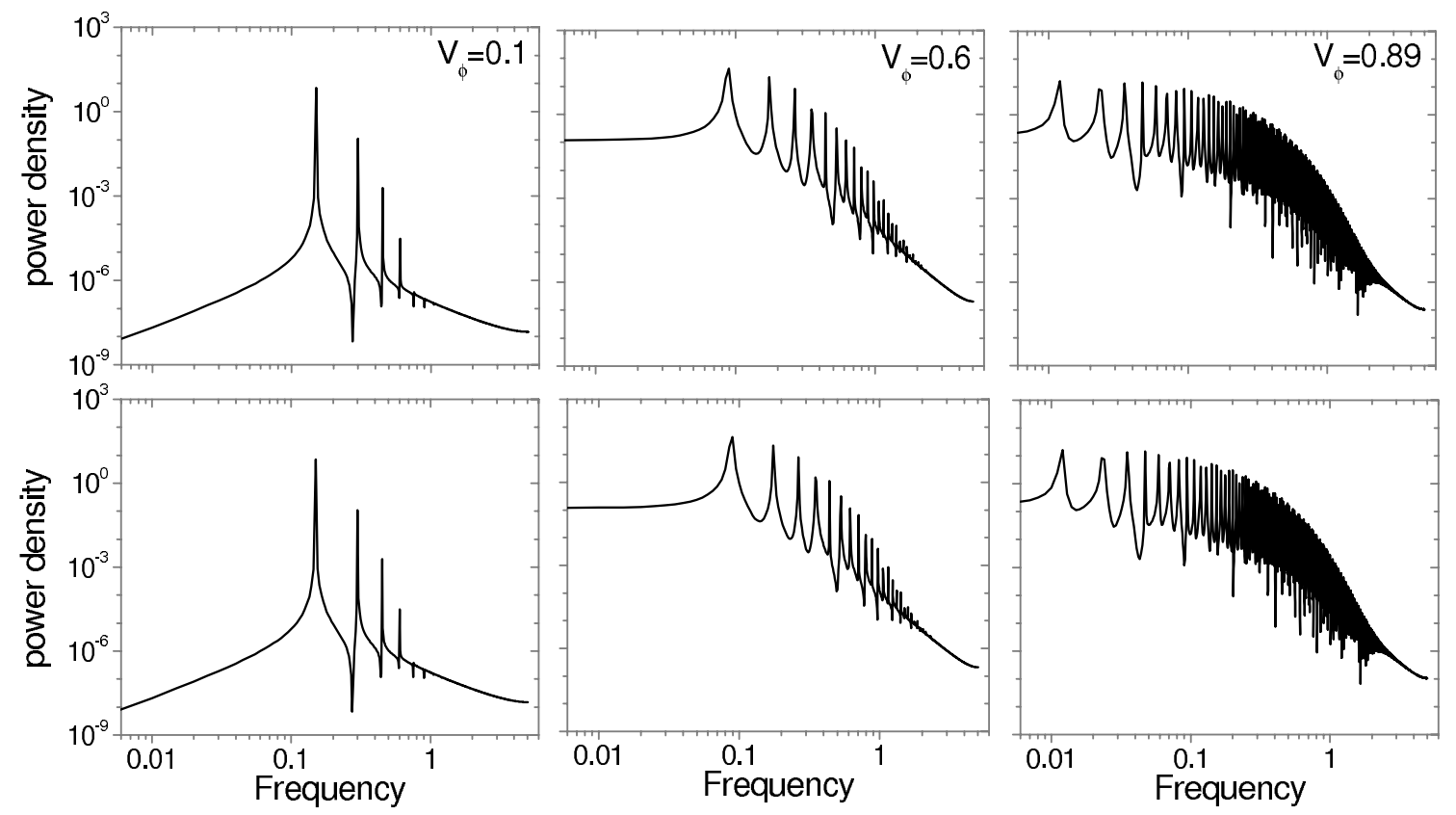

Fig. 3. FFT spectra of both the forward-propagating $E_{\mathrm{i}}$ (upper panels) and the backward-propagating one (lower panels): (1) sinusoidal solitons with $V_{\phi 0}=0.1$ (left-column panels); (2) sawtooth solitons with $V_{\phi 0}=0.6$ (middle-column panels); (3) bipolar solitons with $V_{\phi 0}=$ 0.89 (right-column panels).

function of the only $\Phi$ in advance. It is involved in the coupling of the fluid variables to Maxwell's equations through charge densities $\left(n_{\mathrm{e}}, n_{\mathrm{i}}\right)$ and other components of the flow velocity $\left(\boldsymbol{u}_{\mathrm{i}}\right)$. Numerical calculations reveal that not only no two branches of solitary waves exist in this case, but the three well-known solitary structures are modulated by $V_{\mathrm{z}}$ other than $V_{\phi}$. More significantly, instead of the bipolar shapes, a new type called oscillitons comes into being by solving Eq. (33). This type of nonlinear waves features a relatively LF propagating solitary wave, however, the amplitudes of which are violently modulated by small-amplitude but HF oscillations.

\subsection{Linear modes}

The background linear waves in this case contain three modes in the parallel direction $\left(\theta=0^{\circ}\right)$, depending on $k_{z}$ as given by Eqs. (A7)-(A11). Figure 4 illustrates these background modes in the $\omega-k$ plane with $\xi_{T}=5$ and $\xi_{\omega}=12$.

\subsubsection{Sound wave (SW) mode $\left(k_{z} \ll \xi_{\omega}\right)$}

This mode has a simple dispersion relation as follows: $\left(k_{z} \ll\right.$ $\left.\xi_{\omega}\right)$ :

$\omega^{2}=k_{z}^{2} \zeta$

It reduces to the SSW mode if ions are cold with $\zeta=1$. In this mode, $k_{z}$ is so small as to be neglected compared to $\xi_{\omega}$. A threshold of $1 \%$ is adopted as commonly used in statistics. The corresponding $\omega$ threshold is 0.13 . In the figure with an

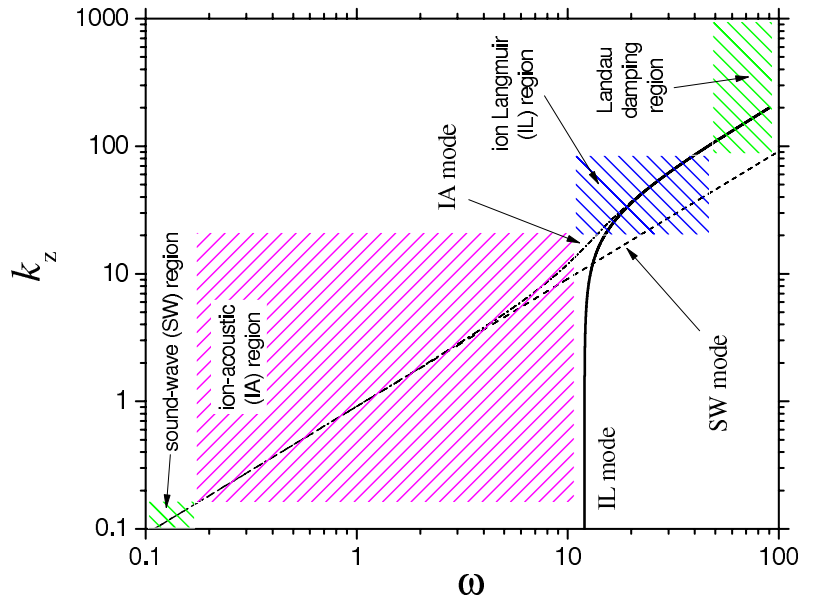

Fig. 4. Background linear oscillation modes in the $\omega-k$ plane with $\xi_{T}=5$ and $\xi_{\omega}=12$.

origin of $(0.1,0.1)$, the region to indicate this mode is labeled roughly (not in scale for an easy identification) by skewed lines on the lower-left corner. Note that all waves with $\omega<$ 0.13 (in a unit of $\Omega_{\mathrm{i}}$ ) are of the SW mode.

\subsubsection{Ion acoustic (IA) mode $\left(k_{z}<x i_{\omega}\right)$}

This mode has

$\omega^{2}=\frac{k_{z}^{2}}{\xi_{T}}+\frac{k_{z}^{2} \xi_{\omega}^{2}}{k_{z}^{2}+\xi_{\omega}^{2}}$ 

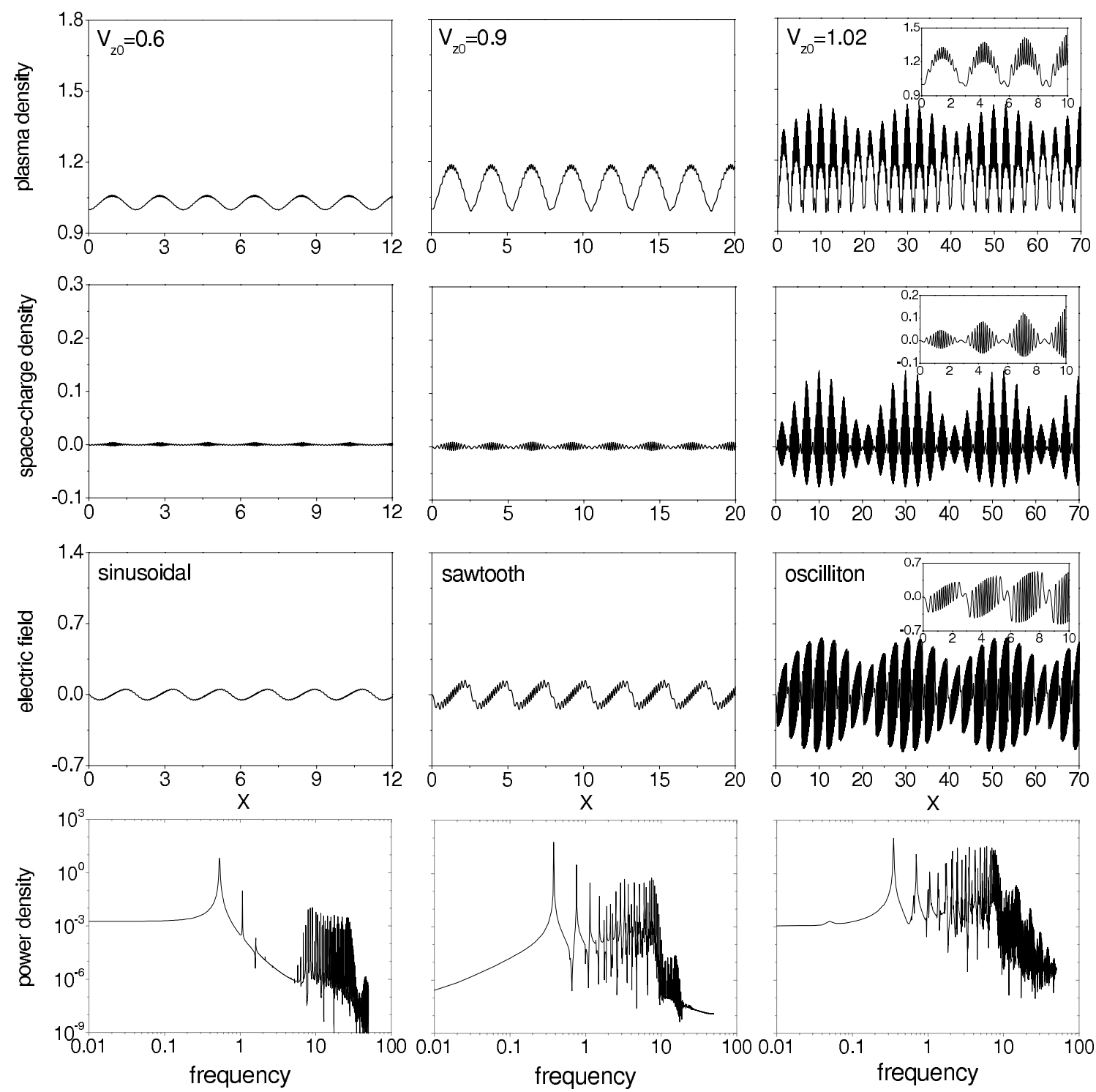

Fig. 5. Three different types of solitary waves under charge non-neutrality conditions with $U_{z 0}=0.6$ (left column), 0.9 (middle column), and 1.02 (right column). Represented by the electric field structures (the third row), the waves are oscillating sinusoidal-shaped (left), oscillating sawtooth-shaped (middle), and oscilliton-shaped (right) shapes. From top to bottom: the plasma density (either $N_{\mathrm{i}}$ or $N_{\mathrm{e}}$ ), space-charge density $\left(N_{\mathrm{sc}}=N_{\mathrm{i}}-N_{\mathrm{e}}\right)$, electric field $\left(E_{\mathrm{i}}\right)$, and FFT power-density spectrum. Input parameters: $M=1.8, R=0.8, \xi_{T}=5$, and $\xi_{\omega}=12$; boundary conditions: $N_{\mathrm{i} 0}=1,\left\{U_{r 0}, U_{\phi 0}\right\}=\{0,0.07,1\}, \Phi_{0}=0$, and $E_{0}=0$.

which occupies a frequency band from below to above ion gyro-oscillations in that $\omega$ is between a frequency around 0.1 and about 10. In the nonlinear regime, a series of harmonics is the signature of this mode, due to the fact that the oscillations are not in the form of pure sine waves, as discussed previously.

\subsubsection{Hybrid IA/IL mode $\left(k_{z} \sim \xi_{\omega}\right)$}

This mode has

$\omega^{2} \sim \xi_{\omega}^{2}\left(\zeta-\frac{1}{2}\right)$
If $k_{z}$ increases to a level close to $\xi_{\omega}, \omega$ is independent of $k_{z}$ but depends only on the ion plasma oscillations as a complete coupling between the IA and IL modes. In view of the spectrum, the high-frequency end of the IA mode should posses a transition region where a narrow-band of noises links the IA harmonic peaks to IL oscillation components.

\subsubsection{Ion Langmuir (IL) $\operatorname{mode}\left(\xi_{\omega}<k_{z}<\sim 10 \xi_{\omega}\right)$}

This mode has

$\omega^{2}=\frac{k_{z}^{2}}{\xi_{T}}+\xi_{\omega}^{2}$ 


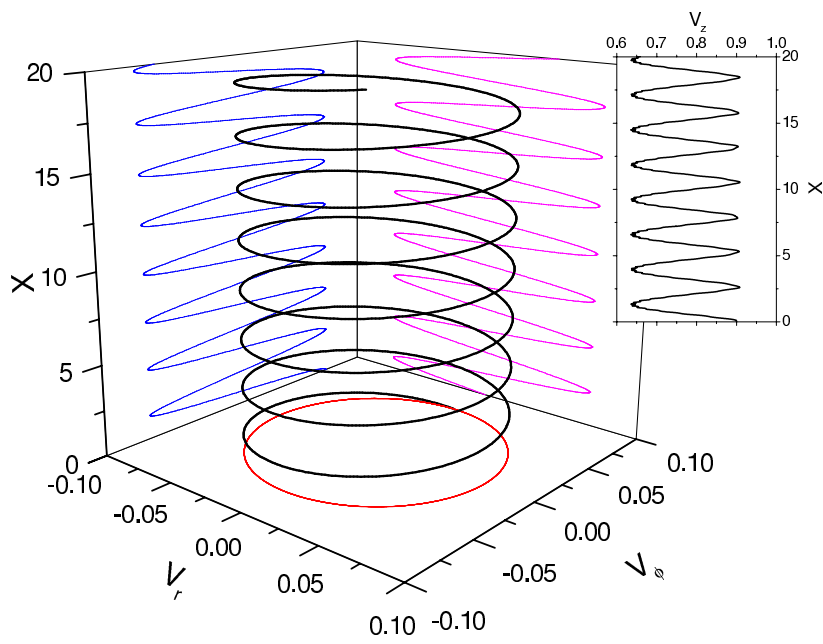

Fig. 6. Features of propagation velocity of a solitary wave under the reference set of data but with $V_{\mathrm{z} 0}=0.9$. In the perpendicular plane in velocity space, $V_{r}$ and $V_{\phi}$ form a helical motion versus $X$, while $V_{\mathrm{Z}}$ has only a unidirectional propagation along $X$ as shown in the inserted panel. All the three velocity components have a common IA period of $X_{1}=2.63$, or, $\omega_{1}=0.3802$.

In this mode, the ion oscillation is at a level of ion plasma frequency; however, $\omega$ relies on the wavelength again, the role of which is increasingly strengthened with $k_{z}$. Although a high-frequency branch as $k_{z}$ becomes very high, the wavelength of the mode is still not so short as to trigger the Landau damping effect. However, after $k_{z} \sim 10 \xi_{\omega}$, or equivalently, $\omega \sim 55$, Eq. (37) begins to provide a phase speed which is in the order of the ion thermal speed. This will initiate the damping effect, another topic beyond the scope of this study. It is thus predictable that the two-fluid system formulated by Eqs. (19)-(23) is unable to produce spectral signatures above $\omega \sim 55$.

\subsection{Structures of solitary waves}

Differing from the previous IA case satisfying charge quasineutrality conditions, there are two more linear modes involved in the initiation and propagation of solitary waves by the introduction of the non-neutrality of charges. One is the lower-frequency SW mode, and the other is the higherfrequency IL mode. The two new modes play ineligible roles through modulations of the IA mode in two aspects: (1) faster and/or slower oscillation ingredients superimposing upon the smoothly-developed amplitudes of the wellknown IA structures; and (2) deformation of these solitary structures into distorted or entirely unexpected shapes.

To show the extraordinary properties of the new solitary waves by solving Eqs. (19)-(23), we continue to use the following reference set of conditions including input parameters: $M=1.8, R=0.8, \xi_{T}=5$, and $\xi_{\omega}=12$; and boundary conditions: $N_{\mathrm{i} 0}=1,\left\{U_{r 0}, U_{\phi 0}, U_{z 0}\right\}=\{0,0.07,1\}, \Phi_{0}=0$, and
$E_{0}=0$. Under $U_{z 0}=0.6,0.9,1.02$, Fig. 5 illustrates modified IA solitary waves. We also use $E_{\mathrm{i}}$ appearances (third row) for classifications. From left to right, the waves are oscillating sinusoidal shocks, sawtooth shocks, and a new type of structures instead of the well-known bipolar shocks, respectively. To get more insight into the features, the plasma density (either $N_{\mathrm{i}}$ or $N_{\mathrm{e}}$; upper row), the space-charge density $\left(N_{\mathrm{sc}}=N_{\mathrm{i}}-N_{\mathrm{e}}\right.$; second row $)$, and the FFT power spectra (bottom row) is exposed.

The first distinctive facet lies in the change of the velocity component to initiate solitary structures. Under previous charge-neutral conditions, it was the azimuthal speed $V_{\phi 0}$ that behaved as the Prime Mover for the three different structures, as illustrated by Figs. 1 and 2. On the other hand, in the present case, $V_{\mathrm{z} 0}$ replaces the role, whereas $V_{\phi 0}$ is united with $V_{r 0}$ to provide initial conditions of a helical trajectory versus $X$ in the perpendicular plane of the velocity space, as illustrated by Fig. 6 with $V_{\mathrm{z} 0}=0.9$, as an example, in which the waveform of the $V_{\mathrm{z}}$ component has been inserted. Numerous calculations indicate that $V_{\mathrm{Z}}$ does not have two branches anymore as before, but is only unidirectional. Note also that the three velocity components expose a common IA period of $X_{1}=2.63$, or, $\omega_{1}=0.38$. This frequency occurs exactly as the dominant peak at 0.38 (the first one from the left) in the FFT spectrum, accompanied by a group of harmonics, in the bottom-middle panel of Fig. 5.

Another striking trait is the oscillation patterns of the amplitudes carried by all three IA structures. The $V_{\mathrm{z} 0}=0.9$ case can be examined again. Through an expanded view of the sawtooth-shaped $E_{\mathrm{i}}$ structure with calculations extending from $X=0$ to 100 , we find that there are two highfrequency modes of small-amplitudes parasitizing inside the low-frequency IA mode: one has longer wavelengths of roughly $X \sim 0.1-0.2$ at the downward edges of the sawteeth, and the other has shorter wavelengths of about $X \sim 0.05-0.1$ at the upward edges. The former falls at $f \sim 5-10$, the hybrid IA/IL band, and the the latter is located at $f \sim 10-50$, the IL band. The FFT panel gives the broadband spectrum from $f=0.01$ to 100 . Clearly, after $f=50$, no power density comes into being. Similar FFT spectra are also found for both sinusoidal and oscilliton-shaped structures. In the sinusoidal case, the hybrid IA/IL band is at $f \sim 8-20$, and the IL band is at $f \sim 20-50$. In the oscilliton mode, the hybrid IA/IL mode shifts into $f \sim 3-7$, and the IL mode occupies a broader band from $f \sim 7$ to 50 . It is worth mentioning here that in the IL band, there are series of harmonic narrow sub-bands peaked at $\sim 7 \pm 2,15 \pm 2,23 \pm 2,31 \pm 2$, $39 \pm 2,46 \pm 2$. Between two adjoint sub-bands noises occur. These sub-band oscillations and noises come from the upward edges of every sawtooth (the inserted panel). It is interesting to note that in the last case when $V_{\mathrm{z} 0}=1.02$, there is a low-frequency SW oscillation at $\sim 0.05$. This oscillation categorizes a new type of soliton structures called as "oscillitons". 

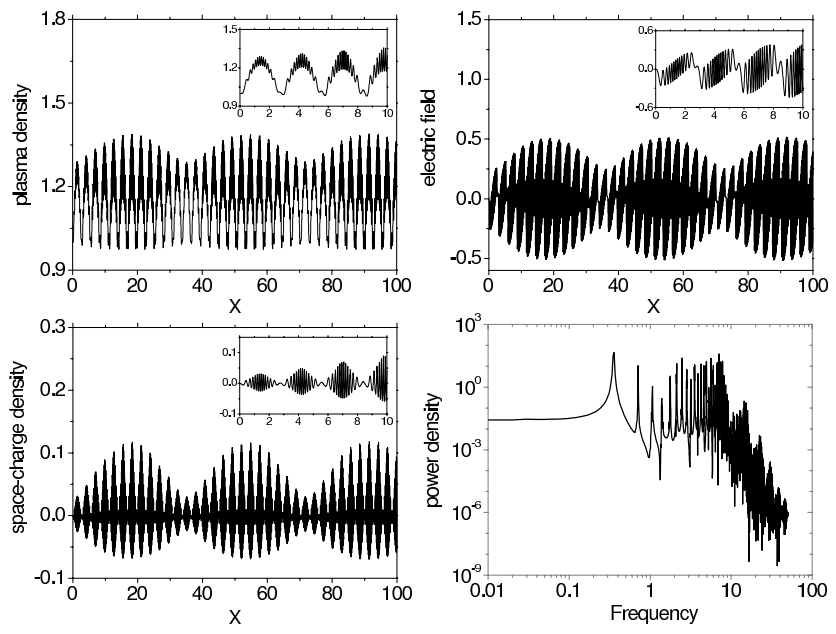

Fig. 7. An example of an oscilliton train under input parameters: $M=1.8, R=0.8, \xi_{T}=5$, and $\xi_{\omega}=12$; and boundary conditions: $N_{\mathrm{i} 0}=1,\left\{U_{r 0}, U_{\phi 0}, U_{z 0}\right\}=\{0,0.07,1\}, \Phi_{0}=0$, and $E_{0}=0$.

This is the third spectacular feature in the two-fluid system under charge non-neutrality conditions. By taking into account the effect of space charges, no bipolar-shaped shocks are present; instead, oscillitons are born. The carrier wave (lowest frequency) is the SW mode, the oscillation of which has the largest wavelength and forms a series of conspicuous clusters of IA packets versus $X$ owing to the periodic changes in amplitudes at a SW frequency; the amplitudes of the carried IA packets vary also periodically, but at an IA frequency. Unexpectedly, through the enlarged plots as given in the inserted panels, these IA packets behave also as carriers loaded with IL oscillations on the upward edges and hybrid IA/IL ones on the downward edges of the sawtooth-like amplitudes. But notice that in the FFT spectrum, the power density of the SW frequency is so weak relative to the background IA mode that only a tiny hump is present at about $f=0.05$. If the SW wavelength is larger, the hump becomes even more unperceptible. See Fig. 7 for an example, a typical case in our calculations. The SW wavelength is $X \sim 35$. At the frequency of $f \sim 0.03$, the corresponding power density is 0.02935 while the background IA power density is 0.02811 , a tiny difference of $4 \%$.

\subsection{Modulations of oscillitons}

The new solitary structures, oscillitons, are featured by HF IA/IL ingredients superimposing upon the LF SW wave. The sweeping band of the spectrum extends from sound-wave frequencies to classical ion plasma frequencies, peaked at IA harmonics and the hybrid IA/IL frequencies. In response to various shapes observed in space plasmas, it is necessary to have a parameterized study on the modulation of the new structures under different conditions.
As introduced in the last section, Fig. 7 is a typical result in simulations. We illustrate plasma density (upper left), space-charge density (lower left), electric field (upper right), and FFT spectrum (lower right) in respective panels, inserted with enlarged solitary structures. By checking data, we know that the plasma density is 1.14 on average, fluctuating from 0.97 to 1.39 . This means the oscillitons are over-densed solitons. They behave as a pump to draw particles from their surroundings. Certainly, close to oscilliton trains, there should exist a transition layer of density dips before reaching the background plasma density.

In addition to the electric and FFT features introduced in the last Sction, the space-charge density varies from $-0.3 \%$ to $11.8 \%$ of the background density $n_{0}$, with an average of $5.2 \times 10^{-6} n_{0}$. Though a very small quantity, it can produce a strong transverse DC electric field $\left(E_{\perp}\right)$ with a characteristic strength of $50-500 \mathrm{mV} / \mathrm{m}$ at a characteristic radius of $10 \mathrm{~m}$ in regions of $n_{0} \sim 10^{11 \sim 12} \mathrm{~m}^{-3}$ (say, ionospheric $F$; Ma and St.-Maurice, 2008). This field is positive and will accelerate surrounding electrons. It should be mentioned that the electric field panel gives the field along $X$, i.e., the parallel electric field of the solitons themselves, not the transverse field mentioned.

\subsubsection{Effect of input parameters}

There are four input parameters in Eqs. (19)-(23): the Mach number $M$, the radial distance from the symmetric axis $R$, the temperature difference between electrons and ions $\xi_{T}$, and the frequency difference between ion plasma oscillations and ion gyrations $\xi_{\omega}$. Figure 8 displays the modulational effect of these parameters on the typical oscillitons shown in Fig. 7 under $M=1.8, R=0.8, \xi_{T}=5$, and $\xi_{\omega}=12$. The left column gives the result at $M=2.8$ when other parameters maintain, while the middle left column is at $R=0.9$, the middle right column is at $\xi_{T}=4$, and the right column is at $\xi_{\omega}=30$, respectively. The plasma density $N_{\mathrm{i}}\left(\right.$ or $\left.N_{\mathrm{e}}\right)$ is illustrated on the top row, while the space-charge density $N_{\mathrm{sc}}$, electric field $E_{\mathrm{i}}$, and FFT power spectra on the second, third, and bottom rows, respectively.

An outstanding feature shown by all the panels is that the solitary structures are sensitive to input parameters. See the left column for an example. Compared to Fig. 7 in which there are roughly three typical SW oscillitons from $X=0$ to 100 , the number is already two in the half $X$ span when $M$ is increased $56 \%$. In contrast, from $X=0$ to 50 when $R$ changes up $12.5 \%$, the middle left column reveal four oscillitons, and the whole train become flattened; when $\xi_{T}$ decreases $20 \%$, the middle right column shows that it is not easy to identify the oscilliton traces from the uneven amplitudes; when $\xi_{\omega}$ goes up by $150 \%$, the right column shows that no oscilliton signatures exist.

Another impressive feature is that the IA and IL modes are present in all cases, as exhibited by the FFT panels on the bottom row: (1) a series of harmonics in the IA band, 

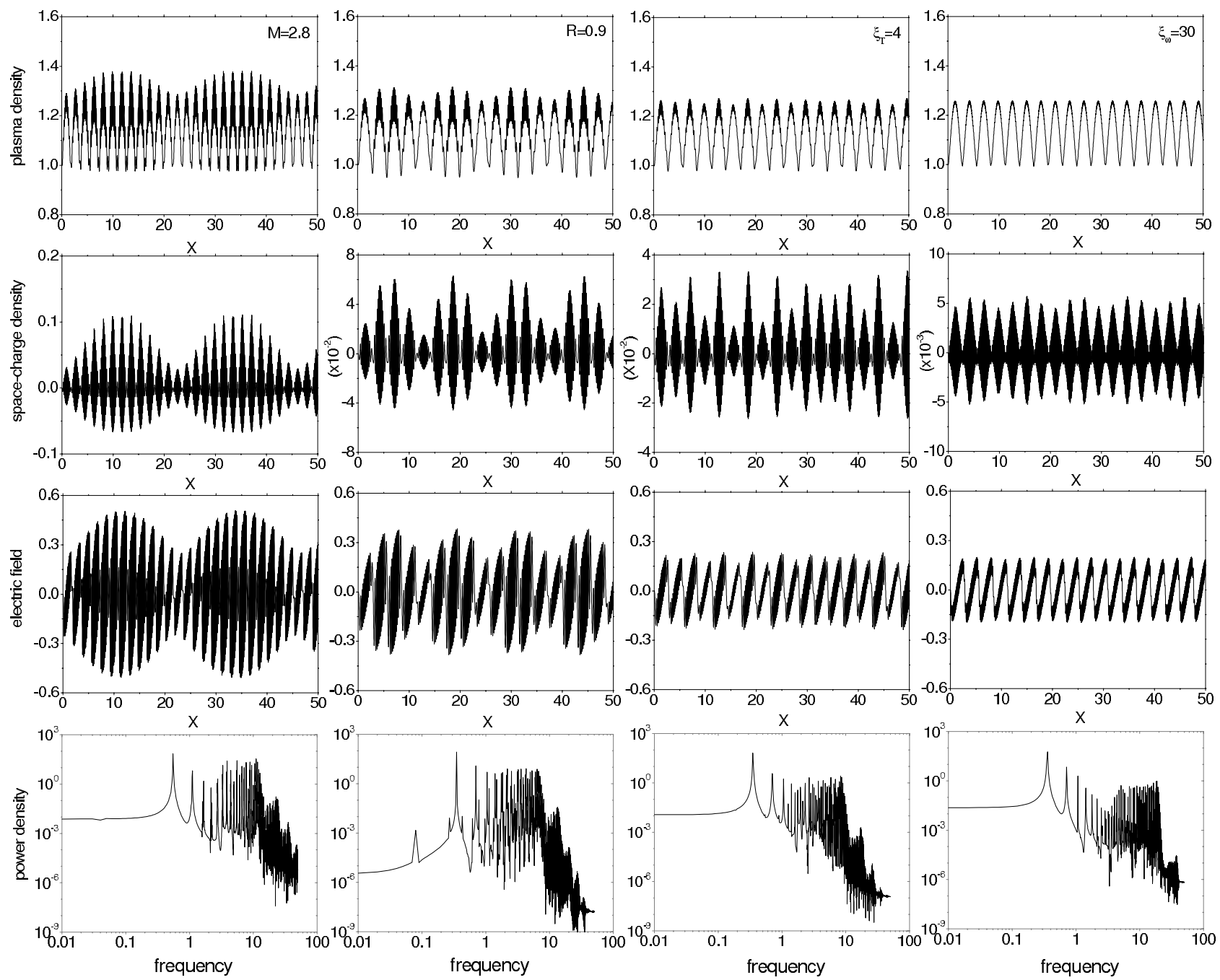

Fig. 8. Modulated oscillitons by input parameters $M$ (left column), $R$ (middle left column), $\xi_{T}$ (middle right column), and $\xi_{\omega}$ (right column), respectively, in view of plasma density (top row), space-charge density (second row), electric field (third row), and FFT power density (bottom row), respectively.

(2) noise peaks in the IA/IL transition region, and (3) a group of harmonic IL narrow bands at the high-frequency end. However, the bands may be shifted more or less from case to case. For instance, the first panel from the left says that the dominant IA peak is at $f \sim 0.55$, while the other three panels provide $\sim 0.35$ or 0.36 . It should be noted here that in the $R$ case, the SW oscilliton peak is evident due to the much weaker background IA power density in the lowfrequency end.

Moreover, whatever a value of an input parameter holds, the plasma density amplitudes and average densities are almost the same in all cases as those in the typical case. For example, the average densities are from 1.13 to 1.14 , and nearly identical to the typical value, 1.13. This means that the solitary waves in all the cases are over-densed. On the contrary, the space-charge density does not have this property. In the typical case, it is $5.2 \times 10^{-6}$, while in Fig. 8, they are $(-8.8,0.8,-1.9,0.7) \times 10^{-6}$, respectively. Consequently, the resultant $E_{\perp}$ are either positive or negative, accelerating or decelerating surrounding ions accordingly.

Last but not least, it is interesting to note that oscilliton structures provide the highest space-charge density and electric field amplitudes. In the typical case, $N_{\mathrm{sc}}$ and $E_{\mathrm{i}}$ of the oscilliton waves are $5.2 \times 10^{-6}$ and 0.5 , respectively. For the non-oscilliton structures in Fig. 8, the space-charge densities mentioned above are all smaller than the typical value; and the electric field amplitudes are $0.35,0.25,0.2$, respectively, also smaller. Clearly, the induced transverse electric field should be the strongest in oscilliton waves.

\subsubsection{Effect of initial conditions}

In real situations, the initial states of space plasmas cannot always be as ideal as to satisfy the conditions required to 

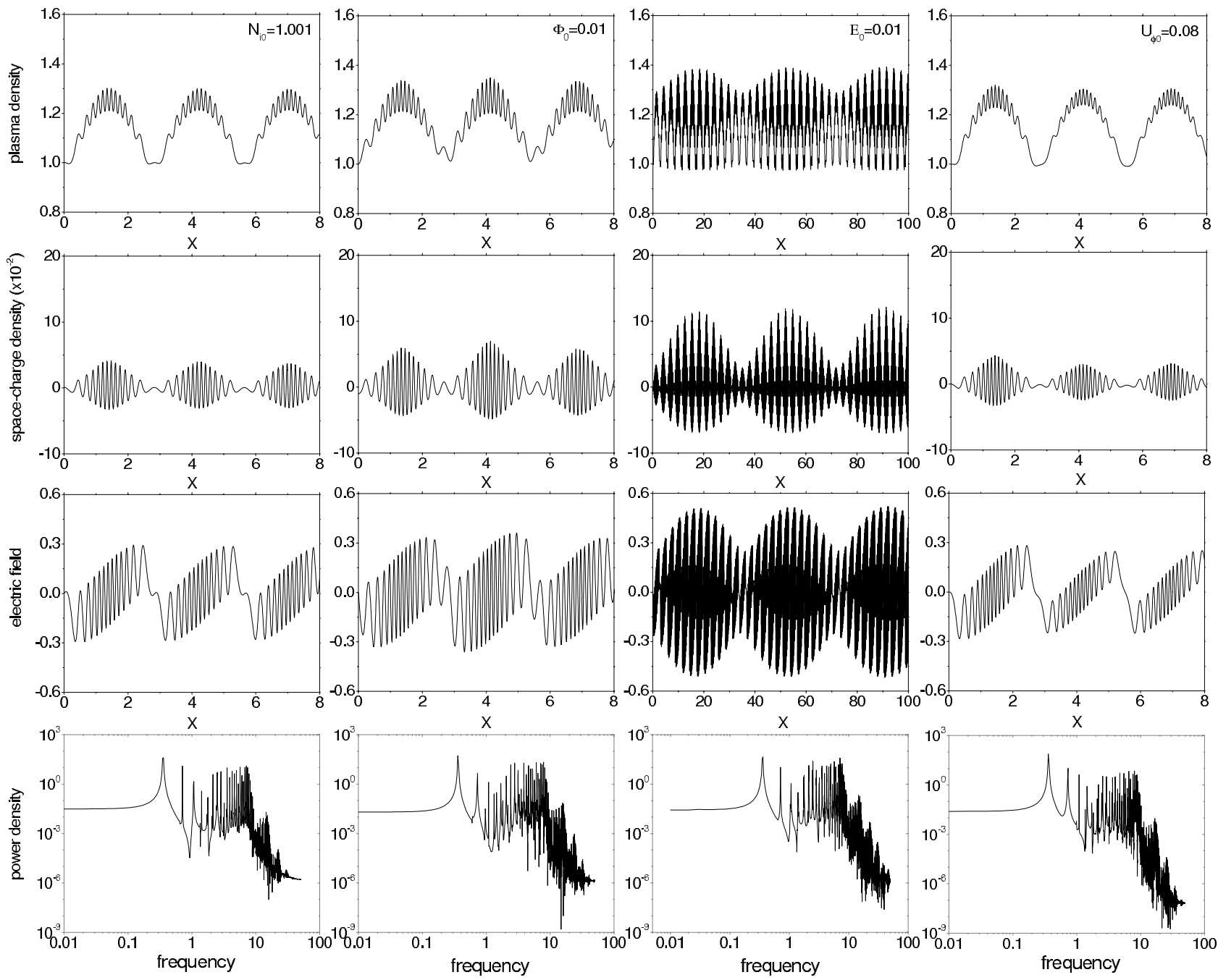

Fig. 9. Same as Fig. 8 but modulated by initial conditions $N_{\mathrm{i} 0}$ (left column), $\Phi_{0}$ (middle left column), $E_{0}$ (middle right column), and $U_{\phi 0}$ (right column), respectively.

drive solitary waves, especially in turbulent plasmas where a small fluctuation of all variables is always occurring because of turbulence. We thus check the effects of a tiny deviation in four parameters $\left(N_{\mathrm{i} 0}, \Phi_{0}, E_{0}\right.$, and $\left.U_{\phi 0}\right)$ from their respective initial states on the typical oscilliton waves given in Fig. 7. We set $N_{\mathrm{i} 0}$ to go up by $0.1 \%, \Phi_{0}$ and $E_{0}$ to increase from 0 to 0.01 , and $U_{\phi 0}$ from 0.07 to 0.08 . Figure 9 displays the results.

First of all, the oscilliton features are found to be highly susceptible to the initial conditions ( $E_{0}$ is an exception, however, changes occur after $\left.E_{0} \sim 0.04\right)$. Though slight changes are made in the initial parameters, the low-frequency SW mode is out of vision; still, the IA and IL modes are left evidently. Only in the $E_{0}$ case, the SW mode is left until $E_{0} \sim 0.05$. After that, no SW periodicity can be identified.

Secondly, just as the input parameters do, initial conditions influence more on $N_{\mathrm{sc}}$ than on $N_{\mathrm{i}}\left(\right.$ or $\left.N_{\mathrm{e}}\right)$. Looking at the first two top rows. the average values of $N_{\mathrm{i}}$ are $1.14,1.17,1.14$, 1.15 , separately, in the four panels, within $3 \%$ of the typical value of 1.14 given in Fig. 7; whereas those of $N_{\mathrm{sc}}$ are (-6.8, $-6.1,6.8,1.44) \times 10^{-6}$, respectively, changing not only in magnitudes up to tens of percent, but also in polarities from positive to negative, relative to the typical average value of $N_{\text {sc }}, 5.2 \times 10^{-6}$.

Lastly, initial conditions regulate wavefield strengths in a way similar to input parameters: whenever lower-frequency ingredients come into being in propagation, a solitary wave will always carry stronger $E_{\mathrm{i}}$ structures. See cases except $N_{\mathrm{i} 0}$ for example. Though $E_{0}$ changes the same as $\Phi_{0}$ and $U_{\phi 0}$ by 0.01 , the solitary wave embodies an additional longerwavelength SW mode, while carrying shorter-wavelength IA/IL solitons just as other waves. It is easy to see that the amplitude of $E_{\mathrm{i}}$ is about 0.5 , higher than other amplitudes. 


\section{Summary and discussion}

In addition to the well-known three types of nonlinear simple waves with single frequencies (namely, sinusoidal, sawtooth, and spiky or bipolar), many high-altitude satellites (e.g., Freja, FAST, Polar, Cluster, and Viking) have reported nonlinear multi-frequency oscillating solitary structures featured by a LF envelope modulated by HF oscillations oscillitons, called "oscillitons" (named by Sauer et al., 2001). Two outstanding studies on them have been performed earlier. One was done by Sauer et al. (2001), who found that in a dusty plasma, the oscillitons can be triggered by the addition of an extra ion population electromagnetically. Later, Kourakis and Shukla (2005) presented a generic methodological formulation for oscillitons in multi-particle systems.

The other study was performed by Verheest et al. (2004). They pointed out that the charge-neutrality condition may be related to the excitation of electromagnetic oscillitons; however, at not too large amplitudes, the oscillitons are very similar to single-frequency solitary envelopes, whether or not the charge neutrality condition is satisfied. Keeping in mind that oscillitons are often reported at high altitudes above ionospheric F-layer, we realized that the formation of oscillitons should be related to the charge-neutrality condition which is not satisfied above the F-layer as much as that below the layer, regardless of either an electromagnetic or electrostatic excitation. We thus focused on a normal two-fluid plasma system containing electrons and singly charged positive ions to investigate the electrostatic excitation of the envelopes of nonlinear oscillitons under a charge non-neutrality condition.

First of all, a warm-up study was introduced by solving a set of nonlinear self-similar two-fluid equations under a charge-neutrality condition. We exhibited features of parallel-propagating, single-frequency IA solitary waves driven in a cylindrically geometry. Three salient shapes of IA solitary waves, proved by bulk of observations and simulations, were reproduced. More details are given in Ma (2010). Then, under a charge non-neutrality condition, we confirmed that not only LF sound wave (SW) oscillations but also HF ion Langmiur (IL) ones are excited in the system. The two new modes superimpose upon the traditional IA carrier and heavily modulate its amplitudes. Therefore, "electrostatic oscilliton" structures are excited. Furthermore, we found that the wavepackets are dependent not only upon input parameters (e.g., the Mach number, the Debye length, and the initial temperature of the particles), but also upon the initial conditions of the system. It can also be noted that the two new modes (SW and IL) are driven by the charge non-neutrality, regardless of frames with which a plasma system is treated. Thus, they also exist in other coordinates. A companion paper will provide results in a Cartesian frame to reveal their similarities and differences with those presented in this paper.

This study concentrated on a two-fluid system in a cylindrical geometry, different from Sauer et al. (2003)'s study where a dusty plasma system is considered in a Cartesian frame for electromagnetic processes. In that case, a magnetic field is also involved in driving nonlinear waves. The authors found that when an extra ion ingredient is added to a twofluid plasma, an additional oscillation is superimposed upon and modulates the amplitudes of simple solitary waves. Interestingly, Verheest et al. (2004) discussed that these HF-LF "symbiotic", electromagnetic nonlinear waves should have some link to the non-neutrality of charged particles, however, at not too large amplitudes, the effect of the charge nonneutrality is difficult to identify.

In the electrostatic regime, by contrast, our recent work in a Cartesian geometry (Ma and Hirose, 2009) disclosed that if the charge neutrality condition is satisfied, conventional single-wave IA solitary waves are driven in three frequently observed structures. On the contrary, if the condition is not met, just as in regions at higher altitudes in geospace, Ma and Hirose (2010) illustrated, in the same geometry and in the presence of the electron inertia, that the multi-frequency "oscilliton" structures are driven, which features an LF solitary envelope in the IC mode, while the HF oscillations come from the lower-hybrid (LH) mode. In addition, the IA constituent, which has a higher frequency but much smaller amplitude, superimposes upon the LH mode.

In the cylindrical geometry, this paper revealed that the non-neutrality leads to two new linear modes (Appendix A), i.e., LF sound wave (SW) mode, and HF ion Langmuir (IL) mode. Besides, the SW mode provides oscilliton packets, the amplitudes of which are violently modulated by the IA oscillations; whereas the upward sides of the IA amplitudes are modulated by the IL oscillations of smaller amplitudes, and, the downward sides of the IA amplitudes are modulated by hybrid IA/IL oscillations. Furthermore, the oscilliton structures are dependent on both input parameters and initial conditions of the system. We also pointed out that the effects of centrifugal and Coriolis forces on the nonlinear features are reflected by the change of the geometric factor, $R$ (the radial distance from the cylindrically symmetric axis). The closer the distance, the stronger the two forces, as exhibited by the two terms, $u_{\phi}^{2} / r$ and $u_{r} u_{\phi} / r$, in Eqs. (3), (4), respectively. Figures 7 and 8 in Sect. 4.3 offer a comparison, when $R$ changes. As already discussed in Ma (2010), these geometric effects are essential in order to exhibit nonlinear simplewave features and explain converging and diverging shocks observed in space plasmas. Our studies in the present paper expose the influence of the two forces on the characteristics of nonlinear oscillitons.

We are discussing nonlinear oscillitons evolved from nonlinear IA solitary waves where the electron inertia is neglected (e.g., Davidson, 1972; Dauxois and Peyrard, 2006). We did not take the inertia (as well as the magnetic field variation) into consideration mainly because we hope to minimize external distractions as much as possible from our focus on the effect of the charge non-neutrality condition. Nevertheless, similar to the charge non-neutrality condition, 
inertia does play an important role for the excitation of oscillitons, as argued already by McKenzie, Verheest, and their co-workers (e.g., McKenzie, 2002; Verheest, 2005, and references therein). In a related paper, Ma and Hirose (2010) illustrated the effect of the electron mass on the formation of nonlinear LH oscillitons, following their work on linear LH waves (Ma and Hirose, 2009). A noticeable result is that the electron mass drives IA/IC solitary waves to evolve into nonlinear LH oscillitons.

Notwithstanding the above with a "warm" two-fluid model in our study (i.e., the model allows for finite electron and ion temperatures which influence the solitary waves through parameter $\xi_{T}$ ), we did not take into consideration the coexistence of a third component, i.e., a "cold" electron population, which was observed in the boundaries of the Earth's magnetosphere. These electrons may also modulate the features of oscillitons. In addition, we did not consider the obliquity effect on oscillitons, i.e., the influence produced by the inclination angle between the propagation direction and $\boldsymbol{B}$. This angle was verified to modulate strongly the profiles of waves (Mamun and Shukla, 2002). We will relax our model by including these important factors in studies to follow. More important, in view of both observations and simulations (see, e.g., Tsurutani et al., 2002), the ponderomotive force produced by the hybrid frequencies existing in oscillitons may result in a more powerful impact on ambient plasma particles than normal solitary waves in the boundary layers of plasma systems. Consequently, the resultant radial ambipolar field may be more efficient to accelerate Maxwellian ions to non-equilibrium states with observable energies as already measured by, e.g., FAST, Cluster, etc. We have introduced the initial results in Ma et al. (2009), and will show the ponderomotive drive in a companion paper.

We would like to finalize this paper by discussing a few interesting issues concerning the two-fluid model used in the text. One question may arise regarding the reasons why a cylindrical geometry has been selected for the model. The motivation to tackle such a geometry is that different scales of space plasmas are observed to have a cylindrical symmetry, similar to fusion plasmas (e.g., Vandenplas and Gould, 1964; Roig and Schoutens, 1986; Date and Shimozuma, 2001; Takahashi et al., 2004). On larger scales, for example, convection vortices about $100-1000 \mathrm{~km}$ in size have been reported and studied by a number of authors (e.g., Vogelsang et al., 1993; Huang et al., 1998). Smaller cylindrical regions are also known to exist in $1-100 \mathrm{~km}$ in diameter, such as auroral rays (e.g., Pietrowski et al., 1999; Danielides and Kozlovsky, 2001). The smallest ionospheric cylindrical structures that we are aware of are described as lower-hybrid cavities, only 10 to a few hundreds of meters in size (e.g., Schuck et al., 2003; Knudsen et al., 2004). The difference between a cylindrical system and a Cartesian one lies in the situation that particles in the former experience extra acceleration through centrifugal and Coriolis forces determined by the geometrical parameter (i.e., the radial distance, $R$; note that the forces becomes zero for $R \rightarrow \infty$, and, the closer the distance from the origin of a cylindrically symmetric flow, the stronger the effects of the centrifugal and Coriolis forces).

Taking these effects into account proved useful in gaining unexpected physical insights into plasma kinetics (e.g., Ma and St.-Maurice, 2008) to explain satellite measurements of co-existing converging and diverging shocks in space (e.g., Ma, 2010). Therefore, being aware of the fact that there have been very few instances where serious attempts were made to investigate the effects of the charge non-neutrality condition on the development of nonlinear processes in a cylindrical geometry, we concentrated on such a subject to see how the geometrical factor $R$ is able to affect the oscilliton structures (see Figs. 7 and 8 and the text for a comparison in Sect. 4.3). It ought to be mentioned here that we assumed homogeneous plasma models in these studies, while we are aware that a cylindrical model may lead to an inhomogeneous density configuration, a consequence as discussed in Ma and St.-Maurice (2008). How much deformation can be produced exactly by this nonuniformity is interesting but beyond the scope of the present study. We will consider this question and answer it in another paper. An initial result was already given in Ma (2009): the nonuniformity is time-dependent, and in periodic oscillations with a quasi-gyro-frequency $\left(\Omega_{\mathrm{q}}\right)$, either or not the charge nonneutrality behaves as a local phenomenon or extends to infinity. This tells us that, at least for much lower-frequency nonlinear structures than $\Omega_{\mathrm{q}}$, the inhomogeneity can still be considered as a "quasi-homogeneity" on temporal averages on different scales.

The second concern is about the fact that the infinitesimally small space-charge density (say, a normalized one, $5.2 \times 10^{-6}$, as supposed in Sect. 4.3.1) can give rise to a bewildering array of spectacularly different nonlinear waveforms (as illustrated in, e.g., Figs. 8 and 9). Here, we provide more clarifications. Since Cole (1971)'s first study, many authors have discussed the behavior of plasma particles in the presence of a "strong" external electric field $(\boldsymbol{E})$, e.g., Schunk and Walker (1972), St.-Maurice et al. (1976), Jørgensen (1978), St.-Maurice and Schunk (1979), Lockwood et al. (1987), Gaimard et al. (1998), see a detailed introduction in Ma (2009). Here, the "strong $\boldsymbol{E}$ " means that the $\boldsymbol{E} \times \boldsymbol{B}$ drift is in the order or higher than the thermal speed of particles, and thus the system deviates more or less from a thermal equilibrium. Many mechanisms can bring about a strong $\boldsymbol{E}$. Such a field existing in space plasmas is usually excited by charge non-neutrality conditions (caused via, e.g., background density perturbations, precipitating electron beams), although the corresponding space-charge density $n_{\text {sc }}$ is so small that it does not break up the charge quasineutrality condition. In th Introduction, we gave an example of a strong characteristic field produced by a small $n_{\mathrm{sc}}$. Reasonably, a slight charge non-neutrality density produces conspicuous consequences, as discussed by e.g., Ma and St.Maurice (2008) which was enlightened by e.g., Cole (1971), 
Winkler et al. (1992), and St.-Maurice et al. (1994). These studies have made us realize that the charge non-neutrality condition should have a similar importance in nonlinear wave propagations. Encouraged by Verheest et al. (2004), we focused on the action of this condition, and finally exhibited its astonishing role played in driving the nonlinear oscilliton strucutures.

Thirdly, we discuss the difference and similarity between the model introduced in this paper and the "Sauer model" explained in, e.g., Sauer et al. (2001, 2003). The former rests in the intrinsical dissimilarity in treating the magnetic field. We introduced an electrostatic model where no oscillating magnetic field components are considered, and obtained nonlinear waves which are purely longitudinal. The reason for choosing this simpler model is that we hoped to exclude other factors in elucidating the impact of the charge nonneutrality condition on the excitation of electrostatic "oscillitons" as much as possible. In contrast, the Sauer model considered electromagnetic processes and described electromagnetic "oscillitons". Notwithstanding their incongruity, the two models provided locally stationary nonlinear solutions which look like each other. This is evident when comparing results by using the ingenious Sauer model (e.g., Sauer et al., 2003) with those illustrated in this paper. In that work, the authors reported that an addition of a second ion population leads to a significant modification to the structure of electromagnetic stationary waves in a single-ion plasma (e.g., Baumgärtel et al., 1997): the single-frequency "bright" or "dark" solitary structures now contain embedded "smallwave" oscillations (see Sect. 4 of the referred paper in details). this new profile, called an "oscilliton", is characterized by a "normal" soliton structure modulated by oscillations, indicating the coexistence of both the classical soliton properties and oscillating phases. Similarly, though using an electrostatic formulation, our model also produces a LF envelope superimposed upon by HF oscillating components internally, while both the envelope and the ingredients appear to constitute one global nonlinear "oscilliton" structure, just as that contributed by Sauer model. By realizing this similarity, we would like to expand the definition of "oscillitons" in following papers by depicting those nonlinear structures composed of HF-LF oscillation components driven either electrostatically or electromagnetically, while being aware that we are excluding pulsations (e.g., Pc1-5) related to linear MHD processes existing in geospace and/or interplanetary spaces.

Last but not least, we have discussed the validity of the two-fluid model itself in studying nonlinear waves. This model has been used for tens of years in nonlinear plasma studies since the very early stage when nonlinear processes became a hot-spot in plasma physics, pioneered by, e.g., Shukla and Yu (1978); Yu et al. (1980), after the BGK work in 1950s (Bernstein et al., 1957). This topic is classical as exhibited by many plasma textbooks (e.g., Bernstein, 1964; Gartenhaus, 1964; Davidson, 1972, 2001; Schunk and Nagy, 2000; Boyd and Sanderson, 2003; Goedbloed and
Poedts, 2004; Bellan, 2006; Dauxois and Peyrard, 2006). From them, we know that the two/multi-fluid model can be adopted widely to describe plasma systems and directly obtain the bulk properties (such as flow speed, temperature, and heat flow), instead of using a kinetic model which needs complicated analytical/numerical solutions for distribution functions of charged particles. Specifically, there are four points to be aware of in applications: (1) the single-fluid MHD model is only dedicated to large-scale systems in both time (i.e., the wave frequency is much less than the gyrofrequency) and space (i.e., the characteristic size is much larger than the Debye length) (see, e.g., Chapter 3 in Goedbloed and Poedts, 2004; Chapter 2 in Bellan, 2006); (2) both the kinetic and the two/multi-fluid models provide details of the plasma properties that can evolve on very short wave lengths (e.g., Debye length, cyclotron radius), and very high frequencies (e.g., electron plasma frequency, cyclotron frequency) (see, e.g., Chapter 2 in Goedbloed and Poedts, 2004; Chapter 12 in Boyd and Sanderson, 2003); (3) the kinetic model and the two/multi-fluid model are equivalent to each other; however, the former is expressed by a microscopic parameter (i.e., the distribution function), and by contrast, the latter deals with macroscopic quantities (see, e.g., Bernstein, 1964; Gartenhaus, 1964); (4) when dealing with physical properties that depend on microscopic details relating not only to spatial and temporal variables, but also to the velocity variable of particles in a fluid element (such as cases where there exist particle trapping, reflection, the Landau damping, or speedrelated collisions, etc.), the kinetic model must be used; otherwise, the simpler, direct two/multi-fluid model provides a much easier tool for use. Note that this model becomes a single-fluid MHD model for large-scale systems (see, e.g., Chapter 7 in Boyd and Sanderson, 2003). It should be noted her that the above descriptions are irrelevant of either electrostatic or electromagnetic cases, in either transverse or parallel directions, no matter the system is either charge neutral or non-neutral.

In planetary atmospheres and ionospheres, the dominant physical property, particle velocity vector $(\boldsymbol{v})$, is an irrotational vector in velocity space, and thus, $\nabla_{\boldsymbol{v}} \cdot \boldsymbol{a}_{\mathrm{s}}=0$ (where $\boldsymbol{a}_{\mathrm{s}}$ is the acceleration vector of a species; see, Schunk and Nagy, 2000). Thus, in collision-free cases or collisional cases where additional properties (such as the collision frequency) are not related to velocity, we can use either the kinetic or two/multi-fluid model to solve problems, as firstly discussed by Grad (1949). In the nonlinear regime, the fluid models have been applied to many fields in addition to plasma physics, such as, solid states, atomic physics, biological processes, etc. (e.g., Dauxois and Peyrard, 2006). To exhibit a double-check, Ma and St-Maurice (2008) applied the kinetic model to the auroral ionospheric F-region under collisionfree conditions, as an example, where extraordinary studies have been done jointly by satellites, rockets, and radars for more than half a century. Then, they demonstrated that all the transport properties were reproduced exactly by a 
two-fluid model (Chapter 6, Ma, 2009), after reproducing the 13-moment transport equations (p. 59, Schunk and Nagy, 2000) from the classical kinetic theory described by Grad (1949). Evidently, the two-fluid model is much simpler than the kinetic model in solving a same problem, and numerous studies have applied the model to solve problems in nonlinear studies (see references of this paper). The work presented here was inspired by the studies with the efficient, two/multi-fluid model in, e.g., Sauer et al. (2001), Verheest et al. (2004), Kourakis and Shukla (2005) in space plasmas in the presence of charge non-neutrality for either electromagnetic cases (e.g., Sauer et al., 2001) or electrostatic ones (e.g., Kourakis and Shukla, 2005). Thus, either from textbooks and also from numerous papers, we can see that it is valid to adopt the fluid model in dealing with plasma systems, if the processes (as considered in the above mentioned model papers, as well as the present manuscript) are not related to microscopic velocity distributions in fluid elements.

We would also like to clarify a doubt about the correlation between the kinetic model and the non-neutrality conditions. As discussed above, the kinetic effects must be taken into account when physical properties are related not only to space and time, but also to velocity (e.g., Chapter 7, Boyd and Sanderson, 2003), regardless of the charge neutrality. Even if a system is completely charge-neutral, we must consider the kinetic effects if physical properties are dependent on microscopic velocity distributions (e.g., Grad, 1949; Bernstein, 1964). An extreme example is the spinodal decomposition in non-ionized binary gases (e.g., Bastea and Lebowitz, 1997), where the system is purely charge neutral, but the kinetic model must be used. By contrast, another extreme example is a non-neutral plasma system (see, e.g., Davidson, 2001). In such a system when all physical properties are independent of speeds, the fluid model is sufficient in applications, although the system is absolutely non-neutral. As a result, there is not a correlation between the kinetic model and the non-neutral condition. They are not coupled with each other.

Finally, thanks to an anonymous referee's important comments, we would like to point out a caveat about the fluid model used in this paper and other contributions from other authors in studying nonlinear plasma waves: under some circumstances, this model may be invalid. For example, for a simple 1-D-case where a parallel potential energy $e \phi(\mathrm{z})$ exist. If it is not too small, i.e., $e \phi(\mathrm{z}) \ll k_{\mathrm{B}} T_{\mathrm{e}}$ is invalid (or, equivalently, $n_{\mathrm{e}} \ll n_{0}$ is not valid in Eq. 1), the parallel distribution function $f$ is a non-Maxwellian. Though the distributions at both ends far away from the origin of the potential structure can be readily described by a Maxwellian function, particles are absent in the central region of the velocity space. At the origin $z=0, f$ is a non-Gaussian, hole-type distribution function, leading to non-linear kinetic effects (such as particle trapping or electron/ion holes) which breaks down the fluid approximation.

In our results, the fluid approximation is valid when the ratio of $n_{\mathrm{e}} / n_{0}$ is nearly constant, like cases in the left panels of Fig. $1\left(V_{0}=0.1\right)$. On the contrary, kinetic effects must be taken into account when this ratio varies a lot, e.g., in the right panels of the figure $\left(V_{0}=0.89\right)$. This can be intuitively understood because the electric field may be strong enough to trap electrons to keep the density profile; or otherwise the profile will be smeared out by the electron thermal motion. This means that there may have particles which can be trapped in the potential well, or, equivalently, not all of which are "passing" ones due to some non-Maxwellian distribution functions. In this case, any departures from a Maxwellian in the presence of the electromagnetic forces should be taken into consideration (see a detailed discussion in, e.g., St.Maurice and Schunk, 1979). Nevertheless, even when the fluid approximation is not reasonably valid, our results provided here can still be considered as a starting point for a kinetic approach to the zeroth-order approximation. Consequently, it becomes important to evaluate the validity of the fluid model in data-fit modelling when non-linear kinetic effects (such as particle trapping or electron/ion holes) cannot be readily omitted.

\section{Appendix A}

\section{Linear wave modes}

The linear IA and IC waves excited in the cylindrical geometry can be obtained by linearizing Eqs. (7)-(11). The dispersion relation is as follows:

$\omega^{2}=k^{2} \frac{\omega^{2}-\cos ^{2} \theta}{\omega^{2}-1}\left(\frac{1}{\xi_{T}}+\frac{\xi_{\omega}^{2}}{k^{2}+\xi_{\omega}^{2}}\right)$

in which $\omega$ and $k$ are the wave frequency and amplitude of the wave vector $(\boldsymbol{k})$, in units of $\Omega_{\mathrm{i}}$ and $\rho_{\mathrm{i}}^{-1}$, respectively; $\theta$ is the inclination angle between $\boldsymbol{k}_{z}$ and $\boldsymbol{k}$, determined by either $k_{r}=k \alpha_{1}\left(\right.$ where $\left.\alpha_{1}=\sin \theta\right)$ or $k_{z}=k \alpha_{2}\left(\right.$ where $\left.\alpha_{2}=\cos \theta\right)$, the radial and axial components of $\boldsymbol{k}$, respectively.

In the perpendicular plane $\left(\theta=90^{\circ}\right)$, Eq. (A1) provides

$\omega^{2}=1+\frac{k_{r}^{2}}{\xi_{T}}+\frac{k_{r}^{2} \xi_{\omega}^{2}}{k_{r}^{2}+\xi_{\omega}^{2}}$

or, in the dimensional form for convenience,

$\omega^{2}=\Omega_{\mathrm{i}}^{2}+k_{r}^{2}\left(v_{\mathrm{T}}^{2}+\frac{c_{\mathrm{s}}^{2}}{k_{r}^{2} / k_{\mathrm{De}}^{2}+1}\right)$

which is evidentally the hybrid IC/IA dispersion relation. It leads to two modes when $k_{r}$ goes to extremes.

On one hand, in the long-wavelength limit, $k_{r} \ll k_{\mathrm{De}}$, it produces a modified IC branch:

$\omega^{2}=\Omega_{\mathrm{i}}^{2}+k_{r}^{2}\left(v_{\mathrm{T}}^{2}+c_{\mathrm{s}}^{2}\right)$

in which the second term appears as a correction to the first term due to the small $k_{r}$. If $k_{r} \ll k_{\mathrm{De}}$, this branch reduces to 
ion gyro-oscillations: $\omega^{2}=\Omega_{\mathrm{i}}^{2}$; for cold ions, it becomes a slow mode:

$\omega^{2}=\Omega_{\mathrm{i}}^{2}+k_{r}^{2} c_{\mathrm{s}}^{2}$

On the other hand, at short wavelengths, $k_{r} \gg k_{\text {De }}$, it evolves to the Upper-hybrid (UH) mode:

$\omega^{2}=\omega_{\mathrm{UH}}^{2}+k_{r}^{2} v_{\mathrm{T}}^{2}$

in which $\omega_{\mathrm{UH}}^{2}=\Omega_{\mathrm{i}}^{2}+\omega_{\mathrm{pi}}^{2}$. If ions are cold, this wave reduces to simple ion upper-hybrid oscillations. At much shorter wavelengths, $k_{r} \gg \gg k_{\mathrm{De}}$, the wave reduces to $\omega^{2}=k_{r}^{2} v_{\mathrm{T}}^{2}$, the group speed of which is comparable to the phase velocity $v_{\mathrm{ph}}=\omega / k_{r}$. The plasma waves can then accelerate ions that are moving with speed in the order of $v_{\mathrm{ph}}$. But the related process often leads to Landau damping and the fluid description used in the present study gives its way to kinetic formulations.

In the parallel direction $\left(\theta=0^{\circ}\right)$, Eq. (A1) gives

$\omega^{2}=k_{z}^{2}\left(\frac{1}{\xi_{T}}+\frac{\xi_{\omega}^{2}}{k_{z}^{2}+\xi_{\omega}^{2}}\right)$

or, in the dimensional form,

$\omega^{2}=k_{z}^{2}\left(v_{\mathrm{T}}^{2}+\frac{k_{\mathrm{De}}^{2}}{k_{z}^{2}+k_{\mathrm{De}}^{2}} c_{\mathrm{s}}^{2}\right)$

Clearly, this is the familiar ion-acoustic (IA) dispersion relation. It leads to two modes under extreme limits of $k_{z}$.

In the long wavelength limit, $k_{z} \ll k_{\text {De }}$, it reproduces the sound-wave (SW) branch:

$\omega^{2}=k_{z}^{2}\left(v_{\mathrm{T}}^{2}+c_{\mathrm{s}}^{2}\right)$

If ions are cold relative to electrons, a slow sound wave (SSW) mode is reached:

$\omega^{2}=k_{z}^{2} c_{\mathrm{s}}^{2}$

In the short wavelength limit, $k_{z} \gg k_{\text {De }}$, it becomes an ion Langmuir (IL) wave with a dispersion like the electron counterpart:

$\omega^{2}=k_{z}^{2} v_{\mathrm{T}}^{2}+\omega_{\mathrm{pi}}^{2}$

If the ions are cold, this wave becomes a simple ion plasma oscillation mode. At shorter wavelengths, $k_{z} \gg \gg k_{\text {De }}$, the waves become

$\omega^{2}=k_{z}^{2} v_{\mathrm{T}}^{2}$

the group speed of which is comparable to the phase velocity $v_{\mathrm{ph}}=\omega / k_{z}$. As discussed above in the perpendicular case, the Landau damping comes into play to break the fluid description. Hence, ion-acoustic waves propagate like sound waves at long wavelengths, whileas they become ion Langmuir waves at short wavelengths.
Acknowledgements. This work was funded by Visiting Fellowships in Canadian Government Laboratories Program, Natural Sciences and Engineering Research Council (NSERC), Canada. J. Ma wishes to thank Frank Verheest and an anonymous referee for their valuable comments which have greatly enhanced the quality of the manuscript.

Topical Editor R. Nakamura thanks F. Verheest and another anonymous referee for their help in evaluating this paper.

\section{References}

Alpert, Ya. L.: Resonance nature of the magnetosphere, Phys. Rep., 339, 323-444, 2001.

Bastea, S. and Lebowitz, J. L.: Spinodal decomposition in binary gases, Phys. Rev. Lett., 78, 3499-3502, 1997.

Baumgärtel, K., Dubinin, E., and Sauer, K.: Story, Solar wind magnetic holes: Signatures of slow mode-type MHD solitons, Adv. Space Res., 20, 69-74, 1997.

Baumjohann, W., Treumann, R., LaBelle, J., and Anderson, R.: Average electric wave spectra across the plasma sheet and their relation to ion bulk speed, J. Geophys. Res., 94, 15221-15230, 1989.

Bellan, P. M.: Fundamentals of plasma physics, Cambridge University Press, Cambridge, 2006.

Bernstein, I. B.: Kinetic theory of gases, the Fokker-Planck equation, transport coefficients in a plasma (Lecture Notes), Princeton University, 1964.

Bernstein, I. B., Green, J. M., and Kruskal, M. D.: Exact non-linear plasma oscillations, Phys. Rev., 108, 546-550, 1957.

Boyd, T. J. M. and Sanderson, J. J.: The physics of plasmas, Cambridge University Press, Cambridge, 2003.

Burchill, J. K., Knudsen, D. J., Bock, B. J. J., Pfaff, R. F., Walis, D. D., Clemmons, J. H., Bounds, S. R., and Stenbaek-Nielsen, H.: Core ion interactions with BBELF, lower hybrid, and Alfvén waves in the high-latitude topside ionosphere, J. Geophys. Res., 109, A01219, doi:10.1029/2003JA010073, 2004.

Cattaert, T. and Verheest, F.: Large amplitude parallel propagating electromagnetic oscillitons, Phys. Plasmas, 12, 012307, doi:10.1063/1.1824038, 2005.

Cattell, C., Mozer, F., Anderson, R., Hones Jr., E., and Sharp, R.: ISEE observations of the plasma sheet boundary, plasma sheet, and neutral sheet, 2. waves, J. Geophys. Res., 91, 5681-5688, 1986.

Cattell, C., Wygant, J., Dombeck, J., Mozer, F. S., Temerin, M., and Russell, C. T.: Observations of large amplitude parallel electric field wave packets at the plasma sheet boundary, Geophys. Res. Lett., 25, 857-860, 1998.

Cattell, C., Dombeck, J., Wygant, J. R., Hudson, M. K., Mozer, F. S., Temerin, M. A., Peterson, W. K., Kletzing, C. A., Russell, C. T., and Pfaff, R. F.: Comparisons of Polar satellite observations of solitary wave velocities in the plasma sheet boundary and the high altitude cusp to those in the auroral zone, Geophys. Res. Lett., 26, 425-428, 1999.

Cattell, C., Neiman, C., Dombeck, J., Crumley, J., Wygant, J., Kletzing, C. A., Peterson, W. K., Mozer, F. S., and André, M.: Large amplitude solitary waves in and near the Earth's magnetosphere, magnetopause and bow shock: Polar and Cluster observations, Nonlin. Processes Geophys., 10, 13-26, doi:10.5194/npg-10-132003, 2003. 
Chatterjee, P. and Roychoudhury, R.: Effect of finite ion temperature on ion acoustic solitary waves in a two-temperature electronplasma system, Can. J. Phys., 75, 337-343, 1997.

Chen, L.-J.: Bernstein-Green-Kruskal electron solitary waves in collisionless plasmas, $\mathrm{PhD}$ thesis, University of Washington, 2002.

Chiueh, T. and Diamond, P. H.: Two-point theory of current-driven, ion-cyclotron turbulence, Phys. Fluids, 29, 76-96, 1986.

Cole, K. D.: Atmospheric excitation and ionization by ions in strong auroral and man-made electric fields, J. Atmos. Terr. Phys., 33, 1241-1249, 1971.

Danielides, M. A. and Kozlovsky, A.: Aurora vortex structures as a result of disturbed geomagnetic conditions, in: The Outer Heliosphere: The Next Frontiers, edited by: Scherer, K., Fichtner, H., Fahr, H. J., and Marsch, E., COSPAR Colloquiua Series, 11, Pergamon Press, Amsterdam, 2001.

Das, G. C., Sarma, J., Gao, Y.-T., and Uberoi, C.: Dynamical behavior of the soliton formation and propagation in magnetized plasma, Phys. Plasmas, 7, 2374-2380, 2000.

Date, H. and Shimozuma, M.: Boltzmann equation description of electron transport in an electric field. with cylindrical or spherical symmetry, Phys. Rev. E, 64, 066410, doi:10.1103/PhysRevE.64.066410, 2001.

Dauxois, T. and Peyrard, M.: Physics of Solitons, Cambridge University Press, Cambridge, 2006.

Davidson, R. C.: Methods in Nonlinear Plasma Theory, Academic Press, New York, 1972.

Davidson, R. C.: Physics of nonneutral plasmas, Imperial College Press, 2001.

De Keyser, J., Dunlop, M. W., Owen, C. J., Sonnerup, B. U. Ö, Haaland, S. E., Vaivads, A., Paschmann, G., Lundin, R., and Rezeau, L.: Magnetopause and Boundary Layer, Space Sci. Rev., 118, 231-320, 2005.

Dovner, P. O., Eriksson, A. I., Boström, R., and Holback, B.: Freja multiprobe observations of electrostatic solitary structures, Geophys. Res. Lett., 21, 1827-1830, 1994.

Dubinin, E., Sauer, K., and McKenzie, J. F.: Solitons and oscillitons in cold bi-ion plasmas: A parameter study, J. Plasma Phys., 68, 27-52, 2002.

Dubinin, E., Sauer, K., and McKenzie, J. F.: Nonlinear stationary whistler waves and whistler solitons (oscillitons). Exact solutions, J. Plasma Phys., 69, 305-330, 2003a.

Dubinin, E., Sauer, K., and McKenzie, J. F.: Solitons, oscillitons and stationary waves in a cold $p-\alpha$ plasma, J. Geophys. Res., 107, 1295, doi:10.1029/2002JA009571, 2003b.

Dubinin, E., Sauer, K., and McKenzie, J. F.: Solitons, oscillitons and stationary waves in a warm $p-\alpha$ plasma, J. Geophys. Res., 107, 1296, doi:10.1029/2002JA009572, 2003c.

Dubinin, E. M., Maksimovic, M., Cornilleau-Wehrlin, N., Fontaine, D., Travnicek, P., Mangeney, A., Alexandrova, O., Sauer, K., Fraenz, M., Dandouras, I., Lucek, E., Fazakerley, A., Balogh, A., and Andre, M.: Coherent whistler emissions in the magnetosphere - Cluster observations, Ann. Geophys., 25, 303-315, doi:10.5194/angeo-25-303-2007, 2007.

Dubouloz, N., Pottelette, R., Malingre, M., Holmgren, G., and Lindqvist, P.: Detailed analysis of broadband electrostatic noise in the dayside auroral zone, J. Geophys. Res., 96, 3565-3579, 1991.

Dupree, T. H.: Theory of phase space density granulation in plasma,
Phys. Fluids, 15, 334-344, 1972.

Earle, G. D., Kelley, M. C., and Ganguli, G.: Large Velocity Shears and Associated Electrostatic Waves and Turbulence in the Auroral F Region, J. Geophys. Res., 94, 15321-15333, 1989.

Eliasson, B. and Shukla, P. K.: Formation and dynamics of coherent structures involving phase-space vortices in plasmas, Phys. Rep., 42, 225-290, 2006.

Ergun, R. E.: Magnetic-field-aligned electric fields associated with Debye-scale plasma structures, Plasma Phys. Control. Fusion, 41, A61-73, 1999.

Ergun, R. E., Carlson, C. W., McFadden, J. P., Mozer, F. S., Delory, G. T., Peria, W., Chaston, C. C., Temerin, M., Elphic, R., Strangeway, R., Pfaff, R., Cattell, C. A., Klumpar, D., Shelley, E., Peterson, W., Moebius, E., and Kistler, L.: FAST satellite observations of electric field structures in the auroral zone, Geophys. Res. Lett., 25, 2025-2028, 1998a.

Ergun, R. E., Carlson, C. W., McFadden, J. P., Mozer, F. S., Delory, G. T., Peria, W., Chaston, C. C., Temerin, M., Roth, I., Muschietti, L., Elphic, R., Strangeway, R., Pfaff, R., Cattell, C. A., Klumpar, D., Shelley, E., Peterson, W., Moebius, E., and Kistler, L.: FAST satellite observations of large-amplitude solitary structures, Geophys. Res. Lett., 25, 2041-2044, 1998b.

Ergun, R. E., Carlson, C. W., McFadden, J. P., and Strangeway, R. J.: Fast auroral snapshot satellite observations of very low frequency saucers, Phys. Plasmas, 10, 454, doi:10.1063/1.1530160, 2003.

Eriksson, A. I., Holback, B., Dovner, P. O., Boström, R., Holmgren, G., André, M., Eliasson, L., and Kintner, P. M.: Freja observatons of correlated small-scale density depletions and enhanced lower hybrid waves, Geophys. Res. Lett., 21, 1843-1846, 1994.

Farrell, W. M., Fitzenreiter, R. J., Kaiser, M. L., Goetz, K., Maksimovic, M., and Reiner, M. J.: Continuum emission and broadband electrostatic noise at the low latitude boundary layer: A diagnostic of boundary layer dynamics, Geophys. Res. Lett., 29, 12, doi:10.1029/2000GL012799, 2002.

Gaimard, P., St.-Maurice, J.-P., Lathuillere, C., and Hubert, D.: On the improvement of analytical calculations of collisional auroral ion velocity distributions using recent Monte Carlo results, J. Geophys. Res., 103, A3, doi:10.1029/97JA03434, 1998.

Gartenhaus, S.: Elements of plasma physics, Rinehart and Winston Inc., 1964.

Goedbloed, H. and Poedts, S.: Principles of Magnetohydrodynamics, with applications to laboratory and astrophysical plasmas, Cambridge University Press, Cambridge, 2004.

Grad, H.: On the kinetic theory of rarefied gases, Comm. Pure Appl. Math., 2, 331-407, 1949.

Gurnett, D.: Plasma wave interactions with energetic ions near the magnetic equator, J. Geophys. Res., 81, 2765-2770, 1976.

Gurnett, D.: Plasma waves and instabilities, in: Collisionless shocks in the heliosphere: Reviews of current research, edited by: Tsurutani, B. and Stone, R. G., Geophys. Monogr., 35, 207 224, 1985.

Gurnett, D. and Frank, L.: Continuum radiation associated with low-energy electrons in the outer radiation zone, J. Geophys. Res., 81, 3875-3885, 1976.

Gurnett, D., Frank, L., and Lepping, R.: Plasma waves in the distant magnetotail, J. Geophys. Res., 81, 6059-6071, 1976.

Gurnett, D., Shawhan, S., and Shaw, R.: Auroral hiss, Z mode radiation, and auroral kilometric radiation in the polar magnetosphere: 
DE 1 observations, J. Geophys. Res., 88, 329-340, 1983.

Heppner, J. P.: Magnetospheric convection patterns inferred from high latitude activity, in: Atmospheric Emissions, edited by: McCormac, B. M. and Omholt, A., Reihold, New York, 251, 1969.

Huang C.-S., Sofko, G. J., McWilliams, K. A., Bristow, W. A., Greenwald, R. A. and Kelley, M. C.: SuperDARN observations of quasi-stationary mesoscale convection vortices in the dayside high-latitude ionosphere, J. Geophys. Res., 103, 29239-29252, 1998.

Hultqvist, B., Christiansen, P. J., Jones, D., Lockwood, M., Moore, T. E., and Saunders, M.: Observations of auroral zone processes by the Viking satellite [and discussion], Philosophical Transactions A (The Royal Society London, mathematical, physical and engineering sciences), 328, 209-220, 1989.

Infeld, E. and Rowlands, G.: Nonlinear waves, solitons, and chaos (2nd edition), Cambridge University Press, Cambridge, 2000.

Jørgensen, L. W.: Calculation of the Ion Velocity Distribution Function in a Rotating Plasma, Jul. Gjellerup. Sølvgade 87, DK-1307 Copenhagen K. Denmark, Risø Report No. 384, 5-26, 1978.

Kivelson, M. G., Khurana, K. K., Walker, R. J., Warnecke, J., Russell, C. T., Linker, J. A., Southwood, D. J., and Polanskey, C.: Io's Interaction with the Plasma Torus: Galileo Magnetometer Report, Science, 274, 396-398, 1996.

Knudsen D. J., Bock, B. J. J., Bounds, S. R., Burchill, J. K., Clemmons, J. H., Curtis, J. D., Eriksson, A. I., Koepke, M. E., Pfaff, R. F., Wallis, D. D., and Whaley, N.: Lower-hybrid cavity density depletions as a result of transverse ion acceleration localized on the gyroradius scale, J. Geophys. Res., 109, A04212, doi:10.1029/2003JA010089, 2004.

Kojima, H., Matsumoto, H., Chikuba, S., Horiyama, S., AshourAbdalla, M., and Anderson, R.: Geotail waveform observations of broadband/narrowband electrostatic noise in the distant tail, J. Geophys. Res., 102, 14439-14455, 1997.

Kourakis, I. and Shukla, P. K.: Exact theory for localized envelope modulated electrostatic wavepackets in space and dusty plasmas, Nonlin. Processes Geophys., 12, 407-423, doi:10.5194/npg-12407-2005, 200.

LaBelle, J., Treumann, R., Haerendel, G., Bauer, O., Paschmann, G., Baumjohann, W., Lühr, H., Anderson, R., Koons, H., and Holzworth, R.: AMPTE IRM observations of waves associated with flux transfer events in the magnetosphere, J. Geophys. Res., 92, 5827-5843, 1987.

Lee, L. C. and Kan, J. R.: Nonlinear ion-acoustic waves and solitons in a magnetized plasma, Phys. Fluids, 24, 430-436, 1981.

Lin, N., Lee, E. S., McFadden, J., Parks, G., Wilber, M., Maksimovic, M., Cornilleau-Wehrlin, N., Fazakarley, A., Lucek, E., Rème, H., Santolik, O., and Zong, Q.-G.: VLF/ELF wave activity in the vicinity of the polar cusp: Cluster observations, Ann. Geophys., 24, 1993-2004, doi:10.5194/angeo-24-19932006, 2006.

Lockwood, M., Bromage, B. J. I., Willis, D. M., Horne, R. B., and St-Maurice, J.-P.: Non-Maxwellian ion velocity distributions observed using EISCAT, Geophys. Res. Lett., 14, 111-114, 1987.

Ma, J. Z. G.: Ion Velocity Distributions in Inhomogeneous and Time-dependent Auroral Situations, PhD thesis, University of Saskatchewan (Canada), 2009.

Ma, J. Z. G.: Nonlinear ion-acoustic (IA) waves driven in a cylindrically symmetric flow. Astrophys. Space Sci., 0004-640X (Print) 1572-946X (Online), 2010.
Ma, J. Z. G. and Hirose, A.: Parallel propagation of ion solitons in magnetic flux tubes, Phys. Scr., 79, 045502, doi:10.1088/00318949/79/04/045502, 2009.

Ma, J. Z. G. and Hirose, A.: Lower-hybrid (LH) oscillitons evolved from ion-acoustic (IA)/ion-cyclotron (IC) solitary waves: effect of electron inertia, Nonlin. Processes Geophys., 17, 245-268, doi:10.5194/npg-17-245-2010, 2010.

Ma, J. Z. G. and St.-Maurice, J.-P.: Ion distribution functions in cylindrically symmetric electric fields in the auroral ionosphere: The collision-free case in a uniformly charged configuration, J. Geophys. Res., 113, A05312, doi:10.1029/2007JA012815, 2008.

Ma, J. Z. G., St-Maurice, J.-P., and Hirose, A.: Non-wave mechanism of transverse ion heating in magnetic flux tubes, Phys. Scr., 80, 025501, doi:10.1088/0031-8949/80/02/025501, 2009.

Mamun, A. A. and Shukla, P. K.: Obliquely propagating electronacoustic solitary waves, Phys. Plasmas, 9, 1474-1477, 2002.

Manfredi, G.: Long-time behavior of nonlinear Landau damping, Phys. Rev. Lett., 79, 2815-2818, 1997.

Matsumoto, H., Kojima, H., Miyatake, T., Omura, Y., Okada, M., Nagano, I., and Tsutsui, M.: Electrostatic solitary waves (ESW) in the magnetotail: BEN wave forms observed by GEOTAIL, Geophys. Res. Lett., 21, 2915-2918, 1994.

Mazelle, C., Winterhalter, D. Sauer, K., Trotignon, J. G., Acuña, M. H., Baumgätel, K., Bertucci, C., Brain, D. A., Brecht, S. H., Delva, M., Dubinin, E., Øieroset, M., and Slavin, J.: Bow shock and upstream phenomena at Mars, Space Sci. Rev., 111, 115181, 2004.

McFadden, J. P., Carlson, C. W., Ergun, R. E., Chaston, C. C., Mozer, F. S., Temerin, M., Klumpar, D. M., Shelley, E. G., Peterson, W. K., Moebius, E., Kistler, L., Elphic, R., Strangeway, R., Cattell, C., and Pfaff, R.: Electron modulation and ion cyclotron waves observed by FAST, Geophys. Res. Lett., 25, 2045-2048, 1998.

McFadden, J. P., Carlson, C. W., Ergun, R. E., Mozer, F. S., Muschietti, L., Roth, I., and Moebius, E.: FAST observations of ion solitary waves, J. Geophys. Res., 108, 8018, doi:10.1029/2002JA009485, 2003.

McKenzie, J. F.: The ion-acoustic soliton: A gas-dynamic viewpoint, Phys. Plasmas, 9, 800-805, 2002.

McKenzie, J. F., Dubinin, E., Sauer, K., and Doyle, T. B.: The application of the constants of motion to nonlinear stationary waves in complex plasmas: a unified fluid dynamic viewpoint, J. Plasma Phys., 70, 431-462, 2004.

Moore, T. E., Chandler, M. O., Pollock, C. J., Reasoner, D. L., Arnoldy, R. L., Austin, B., Kintner, P. M., and Bonnell, J.: Plasma heating and flow in an auroral arc, J. Geophys. Res., 101, 5279-5297, 1996.

Nakamura, Y. and Sugai, H.: Experiments on Ion-acoustic Solitons in a Plasma, Chaos, Solitons Fractals, 7, 1023-1031, 1996.

Pécseli, H. L.: Solitons and weakly nonlinear waves in plasmas, IEEE Trans. Plasma Sci., 13, 53-86, 1985.

Pécseli, H. L., Iranpour, K., Hotter, Ø, Lybekk, B., Holtet, J., Trulsen, J., Eriksson, A., and Holback B.: Lower hybrid wave cavities detected by the FREJA satellite, J. Geophys. Res., 101, 5299-5316, 1996.

Pickett, J. S., Chen, L.-J., Kahler, S. W., Santolík, O., Gurnett, D. A., Tsurutani, B. T., and Balogh, A.: Isolated electrostatic structures observed throughout the Cluster orbit: relationship to magnetic field strength, Ann. Geophys., 22, 2515-2523, 
doi:10.5194/angeo-22-2515-2004, 2004.

Pietrowski, D., Lynch, K. A., Torbert, R. B., Marklund, G., Ivchenko, N., Ranta, A., Danielides, M., and Kelley, M. C.: Multipoint measurements of large DC electric fields and shears in the auroral zone, Geophys. Res. Lett., 26, 3369-3372, 1999.

Pimenta, A. A., Fagundes, P. R., Bittencourt, J. A., Sahai, Y., Gobbi, D., Medeiros, A. F., Taylor, M. J., and Takahashi, H.: Ionospheric plasma bubble zonal drift: a methodology using OI $630 \mathrm{~nm}$ all-sky imaging systems, Adv. Space Res., 27, 12191224, 2001.

Pottelette, R. and Berthomier, M.: Nonlinear electron acoustic structures generated on the high-potential side of a double layer, Nonlin. Processes Geophys., 16, 373-380, doi:10.5194/npg-16373-2009, 2009.

Pottelette, R., Malingre, M., Bahnsen, A., Eliasson, L., Stasiewicz, K., Erlandson, R. E., and Marklund, G.: Viking observations of bursts of intense broadband noise in the source region of auroral kilometric radiation, Ann. Geophys., 6, 573-586, 1988.

Pottelette, R., Ergun, R. E., Treumann, R. A., Berthomier, M., Carlson, C. W., McFadden, J. P., and Roth, I.: Modulated electronacoustic waves in auroral density cavities: FAST observations, Geophys. Res. Lett., 26, 2629-2632, 1999.

Pottelette, R., Treumann, R. A., Berthomier, M., and Jasperse, J.: Electrostatic shock properties inferred from AKR fine structure, Nonlin. Processes Geophys., 10, 87-92, doi:10.5194/npg-10-872003, 2003.

Price, J. C.: Transport equations for dilute plasma in a magnetic field, Phys. Fluids, 16, 90, doi:10.1063/1.1694179, 1973.

Roig, F. S. and Schoutens, J. E.: Remarks on the use of Boltzmann's equation for electrical conduction calculations in metal matrix and in situ composites, J. Mater. Sci., 21, 2767-2770, 1986.

Sagdeev, R. Z. and Galeev, A. A.: Nonlinear Plasma Theory, Benjamin, New York, 1969.

Santolik, O., Gurnett, D. A., Pickett, J. S., Parrot, M., and Cornilleau-Wehrlin, N.: Spatio-temporal structure of storm-time chorus, J. Geophys. Res., 108, 1278, doi:10.1029/2002JA009791, 2003.

Sauer, K., Dubinin, E., and McKenzie, J. F.: New type of soliton in Bi-ion plasmas and possible implications, Geophys. Res. Lett., 28, 3589-3592, 2001.

Sauer, K., Dubinin, E., and McKenzie, J. F.: Wave emission by whistler oscillitons: Application to "coherent lion roars", Geophys. Res. Lett., 29, 2225, doi:10.1029/2002GL015771, 2002.

Sauer, K., Dubinin, E., and McKenzie, J. F.: Solitons and oscillitons in multi-ion space plasmas, Nonlin. Processes Geophys., 10, 121-130, doi:10.5194/npg-10-121-2003, 2003.

Scarf, F., Frank, L., Ackerson, K., and Lepping, R.: Plasma wave turbulence at distant crossings of the plasma sheet boundaries and the neutral sheet, J. Geophys. Res., 1, 189-192, 1974.

Schuck, P. W., Bonnell, J. W., and Kintner Jr., P. M.: A review of lower hybrid solitary structures, IEEE Trans. Plasma Sci., 31, 1125-1177, 2003.

Schunk, R. W. and Nagy, A. F.: Ionospheres: physics, plasma physics, and chemistry, Cambridge University Press, Cambridge, 2000.

Schunk, R. W. and Walker, J. C. G.: Ion velocity distributions in the auroral ionosphere, Planet. Space Sci., 20, 2175-2191, 1972.

Shi, J. K., Xu, B. Y., Torkar, K., and Liu, Z. X.: Nonlinear waves in a low- $\beta$ plasma with cylindrical symmetry, Phys. Plasmas, 8 ,
4780, doi:10.1063/1.1409959, 2001.

Shukla, P. K. and Yu, M. Y.: Exact solitary ion acoustic waves in a magnetoplasma, J. Math. Phys., 19, 2506-2508, 1978.

St.-Maurice, J.-P. and Schunk, R. W.: Auroral ion velocity distributions using a relaxation model, Planet. Space Sci., 21, 11151130, 1973.

St.-Maurice, J.-P. and Schunk, R. W.: Ion velocity distributions in the high latitude ionosphere, Rev. Geophys. Space Phys., 17, 99134, 1979.

St.-Maurice, J.-P., Hanson, W. B., and Walker, J. C. G.: Retarding potential analyzer measurement of the effect of ion-neutral collisions on the ion velocity distribution in the auroral ionosphere, J. Geophys. Res., 81, 5438-5446, 1976.

St.-Maurice, J.-P., Winkler, E., and Hamza, A. M.: Ionospheric ion velocity distributions and associated transport properties in the presence of auroral electric field gradients, J. Geophys. Res., 99, 19527-19548, 1994.

Sydora, R. D., Sauer, K., and Silin, I.: Coherent whistler waves and oscilliton formation: Kinetic simulations, Geophys. Res. Lett., 34, L22105, doi:10.1029/2007GL031839, 2007.

Takahashi, T., Morohashi, K., Iwasawa, N., and Kondoh, Y.: Kinetic simulation for infinitely long cylindrical high-beta plasma with field-null surface, J. Plasma Fusion Res. Series, 6, 485-488, 2004.

Temerin, M., Woldorff, M., and Mozer, F. S.: Nonlinear Steepening of the Electrostatic Ion Cyclotron Wave, Phys. Rev. Lett., 43, 1941-1943, 1979.

Tjulin, A., Eriksson, A. I., and André, M.: Lower hybrid cavities in the inner magnetosphere, Geophys. Res. Lett., 30, 1364, doi:10.1029/2003GL016915, 2003.

Tjulin, A., André, M., Eriksson, A. I., and Maksimovic, M.: Observations of lower hybrid cavities in the inner magnetosphere by the Cluster and Viking satellites, Ann. Geophys., 22, 29612972, doi:10.5194/angeo-22-2961-2004, 2004.

Tsurutani, B. T., Dasgupta, B., Galvan, C., Neugebauer, M., Lakhina, G. S., Arballo, J. K., Winterhalter, D., Goldstein, B. E., and Buti, B.: Phase-steepened Alfvén waves, proton perpendicular energization and the creation of magnetic holes and magnetic decreases: The ponderomotive force, Geophys. Res. Lett., 29, 2233, doi:10.1029/2002GL015652, 2002.

Vaivads, A., André, M., Buchert, S. C., Wahlund, J.-E., Fazakerley, A. N., and Cornilleau-Wehrlin, N.: Cluster observations of lower hybrid turbulence within thin layers at the magnetopause, Geophys. Res. Lett., 31, L03804, doi:10.1029/2003GL018142, 2004.

Vandenplas, P. E. and Gould, R. W.: Equations of a hot inhomogeneous plasma model-I: resonance frequencies of a cylindrical plasma column, Plasma Phys. (J. Nucl. Energy Part C), 6, 449458, 1964.

Verheest, F. and Cattaert, T.: Large amplitude solitary electromagnetic waves in electron-positron plasmas, Phys. Plasmas, 11, 3078-3082, 2004.

Verheest, F. and Cattaert, T.: Parallel propagating electromagnetic solitons and oscillitons in space plasmas and in relativistic electron-positron plasmas, Phys. Scr., T116, 62-66, 2005.

Verheest, F., Cattaert, T., Dubinin, E., Sauer, K., and McKenzie, J. F.: Whistler oscillitons revisited: the role of charge neutrality?, Nonlin. Processes Geophys., 11, 447-452, doi:10.5194/npg-11447-2004, 2004. 
Verheest, F., Cattaert, T., and Hellberg, M. A.: Compressive and Rarefactive Electron-Acoustic Solitons and Double Layers in Space Plasmas, Space Sci. Rev., 121, 299-311, 2005.

Vogelsang, H., Lühr, H., Voelker, H., Woch, J., Bösinger, T., Potemra, T. A., and Lindqvist, P.-A.: An ionospheric traveling convection vortex event observed by ground-based magnetometers and by VIKING, Geophys. Res. Lett., 20, 2343-2346, 1993.
Yu, M. Y., Shukla, P. K., and Bujarbarua, S.: Fully nonlinear ionacoustic solitary waves in a magnetized plasma, Phys. Fluids, 23, 2146-2147, 1980. 